\title{
Coordinated Restoration of Transmission and Distribution System Using Decentralized Scheme
}

Zhao, Jin; Wang, Hongtao ; Liu, Yutian; Wu, Qiuwei; Wang, Zhaoyu; Liu, Yao

Published in:

IEEE Transactions on Power Systems

Publication date:

2020

Document Version

Peer reviewed version

Link back to DTU Orbit

Citation (APA):

Zhao, J., Wang, H., Liu, Y., Wu, Q., Wang, Z., \& Liu, Y. (2020). Coordinated Restoration of Transmission and Distribution System Using Decentralized Scheme. IEEE Transactions on Power Systems, 34(5), 3428 - 3442.

\section{General rights}

Copyright and moral rights for the publications made accessible in the public portal are retained by the authors and/or other copyright owners and it is a condition of accessing publications that users recognise and abide by the legal requirements associated with these rights.

- Users may download and print one copy of any publication from the public portal for the purpose of private study or research.

- You may not further distribute the material or use it for any profit-making activity or commercial gain

- You may freely distribute the URL identifying the publication in the public portal 


\title{
Coordinated Restoration of Transmission and Distribution System Using Decentralized Scheme
}

\author{
Jin Zhao, Student Member, Hongtao Wang, Senior Member, IEEE, Yutian Liu, Senior Member, IEEE, \\ Qiuwei Wu, Senior Member, IEEE, Zhaoyu Wang, Member, IEEE, and Yao Liu
}

\begin{abstract}
This paper proposes a new decentralized restoration scheme in order to realize efficient coordinated restoration of the coupled transmission and distribution (TS-DS) system. First, considering the separated operation of the TS operator (TSO) and DS operators (DSOs) and to avoid the TSO data processing burden, the TS-DS system is divided into several subsystems according to their physical connection. Then, in order to implement the independent but coordinated decision-making of the subsystems, a decentralized decision-making framework is constructed by model decoupling and iterative interaction between TS and DS. Furthermore, for the sake of the convergence of the iterative process with non-convex models, a new decentralized optimization method is developed to obtain the optimal coordinated restoration strategy of the TS-DS system. The proposed decentralized restoration scheme achieves independent decision-making of the TSO and DSOs in the coupled TS-DS system, and solves the non-convergence problem of the decentralized optimization method. The effectiveness of the proposed method is validated using two IEEE standard test systems and a real-world power system.
\end{abstract}

Index Terms-Bulk power system, coordinated restoration, coordination of transmission and distribution systems, power system restoration.

\section{NOMENCLATURE}

\section{A. Indices}

$i \quad$ Index of nodes number

$j \quad$ Index of distribution system number

$l, k, z \quad$ Indices of inner, outer and the third loops

$n, m \quad$ Indices of bus number

\section{B. Sets}

$N_{\mathrm{G}} \quad$ Set of nodes with generation $\left\{1, \ldots, i, \ldots, \mathrm{I}_{\mathrm{G}}\right\}$

$N_{\mathrm{TS}} \quad$ Set of transmission system (TS) buses $\left\{1, \ldots, n, \ldots, \mathrm{N}_{\mathrm{G}}\right\}$

$N_{\mathrm{TL}} \quad$ Set of nodes with transmission loads $\left\{1, \ldots, i, \ldots, \mathrm{I}_{\mathrm{TL}}\right\}$

$N_{\text {DS }} \quad$ Set of distribution system (DS) buses $\left\{1, . ., n, \ldots, \mathrm{N}_{\mathrm{DS}}\right\}$

$N_{\mathrm{DL}} \quad$ Set of nodes with distribution loads $\left\{1, \ldots, i, \ldots, \mathrm{I}_{\mathrm{DL}}\right\}$

$N_{\text {DG }} \quad$ Set of nodes with DGs $\left\{1, \ldots, i, \ldots, \mathrm{I}_{\mathrm{DG}}\right\}$

$N_{\mathrm{RE}} \quad$ Set of RES nodes in the TS $\left\{1, \ldots, i, \ldots, \mathrm{I}_{\mathrm{RE}}\right\}$

Dis $\quad$ Set of DS number $\left\{1, \ldots, j, \ldots, \mathrm{J}_{\mathrm{dis}}\right\}$

This work was supported by the National Key R\&D Program of China (2016YFB0900100), (Corresponding author: Hongtao Wang).

J. Zhao, H. Wang, Y. Liu, and Y. Liu are with the Key Laboratory of Power System Intelligent Dispatch and Control of Ministry of Education (Shandong University), Jinan 250000, China. (e-mail: hizhaojin@163.com; whtwhm@sdu.edu.cn; liuyt@sdu.edu.cn; sduliuyao@163.com).

Q. Wu is with the Center for Electric Power and Energy, Department of Electrical Engineering, Technical University of Denmark, Kgs. Lyngby, DK 2800 (e-mail: qw@elektro.dtu.dk)

Z. Wang is with Iowa State University, Ames, IA 50011, USA (e-mail:wzy@iastate.edu).
$N_{\text {in }} \quad$ Set of power injection buses in DSs $\left\{1, \ldots, n_{\text {in }}, \ldots, \mathrm{N}_{\text {in }}\right\}$

$N_{\text {out }} \quad$ Set of power withdrawal buses in DSs $\left\{1, . ., n_{\text {out }}, \ldots\right.$,

$$
\left.\mathrm{N}_{\text {out }}\right\}
$$

\section{Parameters}

$p_{\mathrm{TL}}, q_{\mathrm{TL}} \quad$ Active, reactive power of transmission load

$p_{\mathrm{DL}}, q_{\mathrm{DL}}$ Active, reactive power of distribution load

$c_{\mathrm{TL}} \quad$ Weighting coefficient of transmission load

$c_{\text {DL }} \quad$ Weighting coefficient of distribution load

$\beta \quad$ Lagrangian multiplier updating parameter

$P_{\mathrm{B}, \max } \quad$ Maximum boundary power between TS and DS

$S_{n m, \max } \quad$ Maximum apparent power of TS line $\mathrm{nm}$

$P_{\mathrm{G}, \max }, P_{G, \min }, P_{G, \text { ini }}$

Maximum, minimum and initial output of generator

$P_{\mathrm{DG}, \max }, P_{D G, \min }$

Maximum and minimum output of DG

$P G_{\mathrm{B}}^{*} \quad$ Response variable with a fixed value

$P D_{\mathrm{B}}^{*} \quad$ Target variable with a fixed value

$\Delta f_{\max } \quad$ Maximum frequency deviation

$V_{\min }, V_{\max }$ Lower and upper limits of voltage

$R \quad$ Ramp rate of generator

$g, b \quad$ Conductance and susceptance of TS line

$r, x \quad$ Resistance and reactance of DS branch

$S \quad$ Capacity of generator

$\varepsilon \quad$ Frequency response rate of generator

$h$ The number of hyperplanes in the cosine polyhedron

$\theta_{\Delta}^{\max } \quad$ The maximum value of the phase angle difference

$d \quad$ Distance between tangent points

D. Variables

$\boldsymbol{x}_{\mathrm{TL}} \quad$ Transmission load pick-up binary variables

$\left[x_{\mathrm{TL}, 1}, \ldots, x_{\mathrm{TL}, i}, \ldots\right]_{1 \times \mathrm{I}_{\mathrm{TL}}} i \in N_{\mathrm{TL}}$

Distribution load pick-up binary variables

$\left[x_{\mathrm{DL}, 1}, \ldots, x_{\mathrm{DL}, i}, \ldots\right]_{1 \times \mathrm{I}_{\mathrm{DL}}} i \in N_{\mathrm{DL}}$

$\boldsymbol{v}, \boldsymbol{w} \quad$ Lagrangian multipliers

$\left[v_{1}, \ldots, v_{j}, \ldots\right]_{1 \times \mathrm{J}}\left[w_{1}, \ldots, w_{j}, \ldots\right]_{1 \times \mathrm{J}} j \in$ Dis

$\boldsymbol{p}_{\mathrm{G}} \quad$ Generators output $\left[p_{\mathrm{G}, 1}, \ldots, p_{\mathrm{G}, i}, \ldots\right]_{1 \times \mathrm{I}_{\mathrm{G}}} i \in N_{\mathrm{G}}$

$\boldsymbol{p}_{\mathrm{RE}} \quad$ Renewable energy sources output

$\left[p_{\mathrm{RE}, 1}, \ldots, p_{\mathrm{RE}, i}, \ldots\right]_{1 \times \mathrm{I}_{\mathrm{RE}}} i \in N_{\mathrm{RE}}$

$\boldsymbol{p}_{\text {DG }}$

DGs output $\left[p_{\mathrm{DG}, 1}, \ldots, p_{\mathrm{DG}, i}, \ldots\right]_{1 \times \mathrm{I}_{\mathrm{DG}}} i \in N_{\mathrm{DG}}$

$\boldsymbol{P}_{\mathrm{B}} \quad$ Boundary power $\left[P_{\mathrm{B}, 1}, \ldots, P_{\mathrm{B}, j}, \ldots\right]_{1 \times \mathrm{J}} j \in$ Dis

$\boldsymbol{P G}_{\mathrm{B}} \quad$ Response variables $\left[P G_{\mathrm{B}, 1}, \ldots, P G_{\mathrm{B}, j}, \ldots\right]_{1 \times \mathrm{J}} j \in D i s$

$P D_{\mathrm{B}} \quad$ Target variable

$p, q \quad$ Active and reactive power of bus

$F \quad$ Objective function 
$T \quad$ Operation time of load pick-up

$f, \Delta f \quad$ Frequency, frequency deviation

$V, \Delta V \quad$ Voltage/voltage deviation

$\delta \quad$ The difference between the target voltage and true value

$P_{n m}, Q_{n m} \quad$ Active and reactive power flow of TS line $\mathrm{nm}$

$S_{n m} \quad$ Apparent power variable of TS line $\mathrm{nm}$

$\theta_{n m} \quad$ Voltage angle difference of bus $n$ and $m$

$\cos ^{*} \theta_{n m} \quad$ Approximation of $\cos \theta_{n m}$

$P_{n}, Q_{n} \quad$ Active and reactive power flow of the $n t h$ DS branch

$L_{n} \quad$ Power loss of the $n t h$ DS branch

\section{INTRODUCTION}

$\mathrm{B}$ ULK power system restoration after complete or partial system collapse is an extensive and far-reaching problem that is essential to grid resilience [1], [2]. According to the North American Electric Reliability Council (NERC) reports, about $72 \%$ of the restoration process was delayed due to coordination problems between different entities in the power system [3]. Therefore, the coordination between different entities is imperative to improve the bulk system restoration efficiency. In the existing practice, the transmission system operator (TSO) is generally responsible for controlling and coordinating restoration strategies after a blackout [4]. In the restoration process, each distribution system (DS) is regarded as a 'passive' load block which waits for power supply from the transmission system (TS), and the distribution system operator (DSO) follows the instructions from the TSO. Being limited by the 'passive' characteristic of the load side, coordinated restoration between the TS and DS has been barely discussed.

Neglecting distribution system operation characteristics has impeded the bulk system restoration. For example, in the Arizona-Southern California Outage in 2011, the influence of the power flow of the DS to TS was not adequately considered, which significantly affected the restoration [5]. On the other hand, the extensive integration of distributed generators (DGs) and demand-side control has converted the 'passive' load side into 'active'. The DS with DGs can provide local power supply to the end-users [6] and send power back to the TS [7], which can facilitate the bulk system restoration. Thus, the coordinated restoration of the TS-DS system needs to be studied.

In recent years, coordination between the TS and DS has attracted increasing attention of several international power grid organizations [8], [9] and independent system operators (ISOs) [10].The coordination of the TS-DS system has been studied in unit commitment [11], risk assessment [7] and economic dispatch problem [12]. In bulk system restoration, the coordination of TS and DS is meaningful in the load restoration stage since DSs are regarded as the load side at the transmission level and this stage can provide a relatively stable voltage and frequency status for the integration of renewable energy sources (RESs). The load restoration in the TS and the operation of the DS are conventionally conducted separately. Simplifying the DS by the on/off status of the TS substation, the load restoration in the TS can be modeled as a mixed integer linear programming (MILP) problem which focuses on maximizing the load pickup amount in the meshed network [13][15]. Regarding the TS as a fixed power supply from the main substation, the operation problems in the DS such as service restoration, outage management and reactive power control, are also transformed into MILP problems in the radial network [6], [16]-[17]. Therefore, a straightforward idea to coordinate the TS-DS system restoration is integrating the common models of TS and DS to form a centralized optimization.

Using the centralized coordination method means all the data of the TS and DSs should be managed by the TSO. It is not applicable because the TSO requires all the parameters of the network, generators and load of the TS and the DSs, while the system data are not fully accessible due to the separately operated TSO and DSOs [11]-[12], [18]. Besides, the centralized method brings a heavy burden for the control center to process data from the whole TS-DS system [11], and the "dispersed, large number and small scale" parameters from the DS level are difficult to be centrally controlled at the TS level [7], [19]. Therefore, the key to achieving efficient coordinated restoration of the TS-DS system is performing a decentralized restoration scheme. The goal of the decentralized scheme is to obtain a decentralized restoration strategy in the TS-DS system. The optimal decentralized restoration strategy depends on the decomposition coordination optimization method. Several distributed or decentralized algorithms are summarized in [20] to realize decomposed coordination in electric power systems. Generally, these methods can be classified into three types: i) the Lagrangian relaxation based methods such as the analytical target cascading (ATC) [11], [21], alternating direction method of multipliers (ADMM) [22] and auxiliary problem principle (APP) [23]; ii) the Karush-Kuhn-Tucker (KKT) conditions based methods such as optimality condition decomposition (OCD) [24] and heterogeneous decomposition (HGD) [12] and iii) the benders decomposition (BD) method [18], [25]. To realize decentralized calculation in the TS-DS system, the ATC method was used in [11] to solve the coordinated unit commitment problem. Ref. [12] proposed an HGD algorithm to deal with the transmission and distribution coordinated economic dispatch problem. Focusing on voltage problems in the integrated transmission and distribution networks, ref. [18] employed the BD to obtain decentralized reactive power optimization. Among these methods, the ATC is a model-based method for multilevel hierarchical optimization problems and does not require convexity of the model [20][21], which makes it attractive to solve the TS-DS system restoration problem. However, in the TS and DS AC power flow calculation, tremendous discrete variables and special constraints in the restoration model cause non-convergence issues for the ATC method. Thus, the ATC method should be improved to solve the decentralized restoration optimization problem.

This paper proposes a decentralized restoration scheme for efficient coordinated restoration of the TS-DS system. Firstly, the TS-DS system is divided into subsystems. Based on the subsystems, the coupled models of the TS and DSs are pre- 
sented. Secondly, in order to achieve decentralized decisionmaking, the optimization models of the TSO and DSOs are decoupled and an interactive iteration between the TS and DSs is designed based on the ATC method. Finally, to further deal with the non-convergence issue of the ATC method with the non-convex restoration model, a new three loop ATC (TL_ATC) method is proposed to simplify decentralized calculation models and provide the optimal decentralized restoration strategy.

The contributions of this paper are threefold. 1) The bulk power system restoration considering the operation of DSs is proposed and realized by the TS and DS coordinated restoration. Thus, the security problems caused by neglecting DS operation characteristics can be avoided. 2) The proposed coordinated restoration uses power supplies at different voltage levels such that it can perform the conventional "top-down" and "bottom-up" restoration idea in parallel [26]-[28]. As such, the restoration process is accelerated. 3) The TL_ATC method is developed by improving the ATC method. The TL_ATC method guarantees the convergence of the decentralized optimization with non-convex models and simplifies the iterative models of the ATC method. Thus, the TL_ATC method is with better computation performance and can be seamlessly integrated into the existing models of TS restoration and DS operation.

The rest of the paper is organized as follows. Section II describes the problem of the TS-DS system restoration. The realization of decentralized decision-making is introduced in Section III. Section IV presents the TL_ATC method to obtain the decentralized restoration strategy. Section V provides the case studies, followed by conclusions.

\section{Structure of The TS-DS System AND The Coupled MODEL OF COORDINATED RESTORATION}

This section introduces the TS-DS system restoration. The whole system is divided into subsystems, and the centralized model is transformed into coupled models of TSO and DSOs.

\section{A. Hierarchical and Partitioned Subsystems of the TS-DS system}

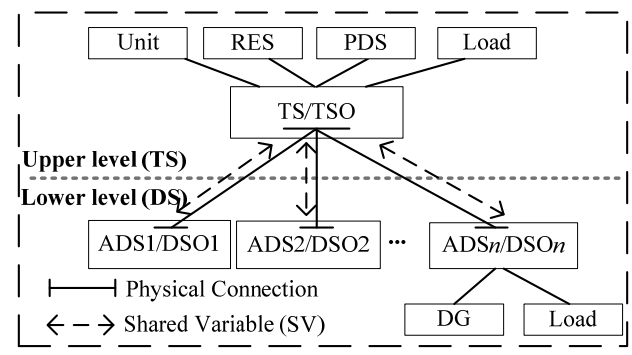

Fig. 1. Structure of the TS-DSs restoration system.

Similar to the concept of 'system of systems' [11], the TSDS system contains multi-level grids and various elements. The conventional TS consists of traditional generators, loads directly connected to the transmission network and passive DSs (PDS). With renewable energy integrated, RESs, such as large-scale wind farms or solar systems, become new elements of the TS. In the DS, the active distribution system (ADS) is formed with flexible demands and DGs. The two-way communication infrastructures are installed in the TS-DS system to provide bidirectional information exchange between the TSO and DSO [8]. In the restoration process, the TSO and DSO are two kinds of system regulators. As shown in Fig. 1, the TS and DS are physically connected through feeders at substations, and a hierarchical upper-lower structure is determined according to the voltage level of power grids. The TSO has the leading responsibility for system restoration, whereas DSOs manage their own networks [29].

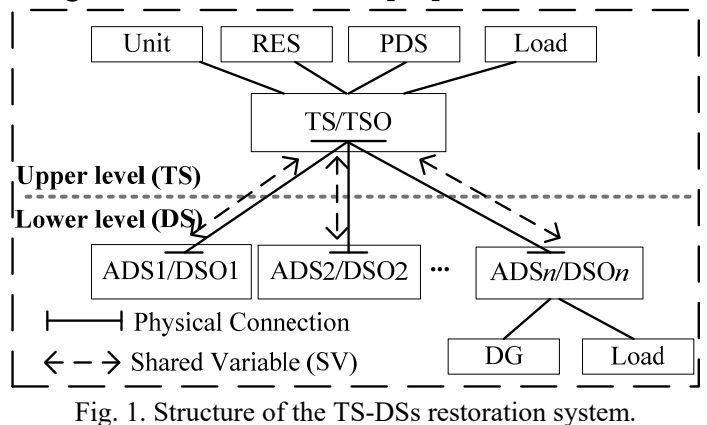

For the system in Fig. 1, the key to dividing the TS-DS system is the physical connection between the TS and DS. As Fig. 2 shows, the power exchange at the boundary bus between the TS and DS is defined as a shared variable (SV). Then, the SV in the TS can be regarded as a pseudo load with power withdrawal while the SV in the DS is a pseudo generator with power injection. Since there is extensive integration of DGs in the DS, the pseudo power withdrawal/ injection may be negative if power transmits from the DS to the TS. With SVs and the separate spatial distribution of DSs, the TS-DS system is divided into subsystems. As the TSO has leading responsibility for system restoration, subsystem 0 belongs to the upper level. Subsystems 1- $n$ refer to different parts which are managed by DSO1-DSO $n$ at the lower level.

\section{B. Coupled Restoration Models Based on Subsystems}

According to [2], the enhancement of power system resilience in the restoration procedure refers to the rapid completion of the restoration process. Since DSs are load clusters, the TS-DS coordination is applied in the load restoration stage which focuses on large amounts of loads recovery. Hence, the objective of the TS-DS coordinated restoration is to pick up load as much and rapid as possible. The generators have been restarted [14], and they are ramping up with a fixed ramp rate [30].

$$
\begin{aligned}
& \operatorname{Max}\left(\begin{array}{l}
\sum_{i \in N_{\mathrm{TL}}}\left(c_{\mathrm{TL}, i} p_{\mathrm{TL}, i} x_{\mathrm{TL}, i}\right) \\
+\sum_{j \in D i s} \sum_{i \in N_{\mathrm{DL}, j}}\left(c_{\mathrm{DL}, j, i} p_{\mathrm{DL}, j, i} x_{\mathrm{DL}, j, i}\right)
\end{array}\right)-\sum_{i \in G} f_{i}(T) \\
& \text { s.t. } \boldsymbol{g}\left(\boldsymbol{x}_{\mathrm{TL}}, \boldsymbol{p}_{\mathrm{G}}, \boldsymbol{p}_{\mathrm{RE}}, \boldsymbol{P}_{\mathrm{B}}, \boldsymbol{x}_{\mathrm{DL}}, \boldsymbol{p}_{\mathrm{DG}}, T\right) \leq 0 \\
& \boldsymbol{h}\left(\boldsymbol{x}_{\mathrm{TL}}, \boldsymbol{p}_{\mathrm{G}}, \boldsymbol{p}_{\mathrm{RE}}, \boldsymbol{P}_{\mathrm{B}}, \boldsymbol{x}_{\mathrm{DL}}, \boldsymbol{p}_{\mathrm{DG}}, T\right)=0
\end{aligned}
$$

The centralized optimization model of the whole system restoration is described by (1)-(3). The objective is to maximize load recovery amount which contains loads $p_{\mathrm{TL}}$ in the TS and loads $p_{\mathrm{DL}}$ in the DS, and minimize the load pickup/operation time $T$. The decision variables are binary 
variables of TS and DS load pickup status $\boldsymbol{x}_{\mathrm{TL}}$ and $\boldsymbol{x}_{\mathrm{DL}}$, load pickup time $T$, the power support $\boldsymbol{p}_{\text {RES }}$ from RESs in TS level and $\boldsymbol{p}_{\mathrm{DG}}$ from DGs in DS level. The SV vector is $\boldsymbol{P}_{\mathrm{B}}$ which contains the boundary powers between the TS and DSs. $\boldsymbol{p}_{\mathbf{G}}$ is the generator output vector which is proportional to the operation time in each step. Function $f()$ represents the relationship between $T$ and generator output. $g()$ and $h()$ are all the equality and inequality constraints which contain power supply constraints, AC power flow related constraints, security constraints and boundary power limitations in the TS-DS system.

The optimization of Model (1)-(3) is impractical because of the information privacy issue of the subsystems and the heavy data processing burden of the TS-DS united optimization for the TSO. Thus, based on the structure of the TS-DS system in Fig. 1, all the variables and constraints in Model (1)-(3) are divided for the TS and each DS. Through replacing each $P_{\mathrm{B}}$ by a TS related response variable $P_{\mathrm{GB}}$ and a DS related target variable $P_{\mathrm{DB}}$, the optimization Model (1)-(3) can be rewritten as (4)-(9). In Model (4)-(9), the constrains and the decision variables are separated according to the subsystems in Fig. 2. The inequality and equality constraints (2) and (3) are divided into (5) and (6) of the TS and (7) and (8) of the $j$ th DS, and so do the decision variables. In the model, the subsystems are coupled by $\boldsymbol{\sigma}$.

$$
\begin{array}{ll}
\operatorname{Max} & \left(\begin{array}{l}
\sum_{i \in N_{\mathrm{TL}}}\left(c_{\mathrm{TL}, i} p_{\mathrm{TL}, i} x_{\mathrm{TL}, i}\right) \\
+\sum_{j \in D i s} \sum_{i \in N_{\mathrm{DL}, j}}\left(c_{\mathrm{DL}, j, i} p_{\mathrm{DL}, j, i} x_{\mathrm{DL}, j, i}\right)
\end{array}\right)-\sum_{i \in N_{\mathrm{G}}} f_{i}(T) \\
\text { s.t. } \quad & \boldsymbol{g}_{\mathrm{TS}}\left(\boldsymbol{x}_{\mathrm{TL}}, \boldsymbol{p}_{\mathrm{G}}, \boldsymbol{p}_{\mathrm{RE}}, \boldsymbol{P} \boldsymbol{G}_{\mathrm{B}}, T\right) \leq 0 \\
& \boldsymbol{h}_{\mathrm{TS}}\left(\boldsymbol{x}_{\mathrm{TL}}, \boldsymbol{p}_{\mathrm{G}}, \boldsymbol{p}_{\mathrm{RE}}, \boldsymbol{P} \boldsymbol{G}_{\mathrm{B}}, T\right)=0 \\
& \boldsymbol{g}_{\mathrm{DS}, j}\left(\boldsymbol{x}_{\mathrm{DL}, j}, \boldsymbol{p}_{\mathrm{DG}, j}, P D_{\mathrm{B}, j}\right) \leq 0 \quad \forall j \in D i s \\
& \boldsymbol{h}_{\mathrm{DS}, j}\left(\boldsymbol{x}_{\mathrm{DL}, j}, \boldsymbol{p}_{\mathrm{DG}, j}, P D_{\mathrm{B}, j}\right)=0 \quad \forall j \in D i s \\
& \sigma_{j}=P G_{\mathrm{B}, j}-P D_{\mathrm{B}, j}=0 \quad \forall j \in D i s
\end{array}
$$

Based on the Lagrangian decomposition method [31], the constraint set $\boldsymbol{\sigma}=\left[\sigma_{1}, \sigma_{2} \ldots \sigma_{j} \ldots\right]=0$ is transformed into $\Phi(\boldsymbol{\sigma})$ in objectives, and Model (4)-(9) is further transformed into a TSO optimization model (10)-(12) at the upper level and several DSO optimization models (13)-(15) at the lower level. Models (10)-(15) correspond to the subsystems in Fig.2.

$$
\begin{aligned}
\text { upper level: } \operatorname{Max} & \sum_{i \in N_{\mathrm{TL}}}\left(c_{\mathrm{TL}, i} p_{\mathrm{TL}, i} x_{\mathrm{TL}, i}\right)-\sum_{i \in N_{\mathrm{G}}} f_{i}(T)+\Phi(\boldsymbol{\sigma})( \\
\text { s.t. } \quad & \boldsymbol{g}_{\mathrm{TS}}\left(\boldsymbol{x}_{\mathrm{TL}}, \boldsymbol{p}_{\mathrm{G}}, \boldsymbol{p}_{\mathrm{RE}}, \boldsymbol{P} \boldsymbol{G}_{\mathrm{B}}, T\right) \leq 0 \\
& \boldsymbol{h}_{\mathrm{TS}}\left(\boldsymbol{x}_{\mathrm{TL}}, \boldsymbol{p}_{\mathrm{G}}, \boldsymbol{p}_{\mathrm{RE}}, \boldsymbol{P} \boldsymbol{G}_{\mathrm{B}}, T\right)=0 \\
\text { lowerlevel : } \operatorname{Max} & \sum_{i \in N_{\mathrm{DL}, j}}\left(c_{\mathrm{DL}, j, i} p_{\mathrm{DL}, j, i} x_{\mathrm{DL}, j, i}\right)+\Phi(\boldsymbol{\sigma}) \\
\text { s.t. } \quad & \boldsymbol{g}_{\mathrm{DS}, j}\left(\boldsymbol{x}_{\mathrm{DL}}, \boldsymbol{p}_{\mathrm{DG}, j}, P D_{\mathrm{B}, j}\right) \leq 0 j \in \text { Dis } \\
& \boldsymbol{h}_{\mathrm{DS}, j}\left(\boldsymbol{x}_{\mathrm{DL}}, \boldsymbol{p}_{\mathrm{DG}, j}, P D_{\mathrm{B}, j}\right)=0 \quad j \in \text { Dis }
\end{aligned}
$$

Note that the connection between subsystems are still maintained since the non-separable penalty function $\Phi(\boldsymbol{\sigma})$ in (10) and (13) depends on more than one subsystem. Despite in a decentralized form, the optimization models are still coupled by the penalty function $\Phi(\boldsymbol{\sigma})$. The coupling prevents the TSO and each DSO making decisions independently based on their own operation and control regulations.

\section{DeCentralized DeCISION-MAKING FrameWORK For COORDINATED RESTORATION}

This section introduces the decentralized decision-making framework constructed by model decoupling and iterative interaction process. The model decoupling allows coordinating the entities within TSO and each DSO by independent optimization. The coordination between the TSO and each DSO is achieved by the iterative interaction process.

\section{A. Decoupled Restoration Optimization Models of TSO and DSOs}

The augmented Lagrangian method with a quadratic penalty term (5) is used to relax the constraints set $\boldsymbol{\sigma}$ in model (2) [32].

$$
\Phi(\boldsymbol{\sigma})=\boldsymbol{v} \boldsymbol{\sigma}^{\mathrm{T}}+\left\|\boldsymbol{w} \circ \boldsymbol{\sigma}^{\mathrm{T}}\right\|_{2}^{2}=\sum_{j \in D i s}\left(v_{j} \sigma_{j}+\left\|w_{j} \circ \sigma_{j}\right\|_{2}^{2}\right)
$$

where $\boldsymbol{v}$ is a Lagrangian multiplier parameter vector while $\boldsymbol{w}$ is a vector of the quadratic term. These two vectors are penalty multipliers of model decoupling and are updated during the iterative process, which is discussed in Subsection $B$. The symbol $\circ$ represents the Hadamard product which is an entrywise multiplication of two vectors.

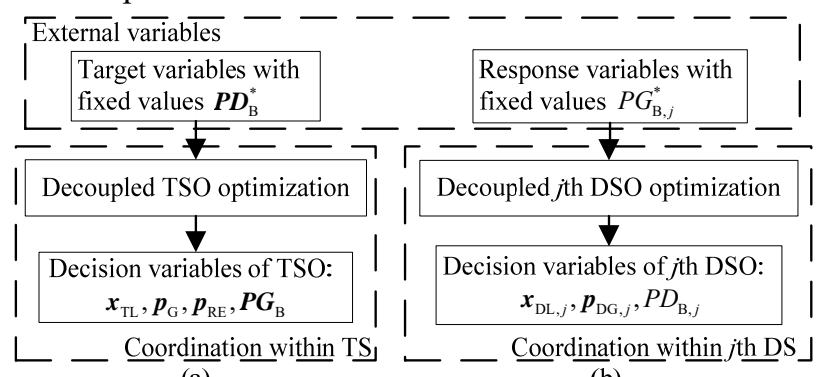
(a)

(b)

Fig. 3. Coordination within TS (a) and coordination within each DS (b)

Since $\sigma$ means the distance between the response variable $P G_{\mathrm{B}}$ and target variable $P D_{\mathrm{B}}$, the target variables are the only external variables for the TSO optimization model (10)-(13) while the response variable is the only unmanaged one for each DSO model (13)-(15). Therefore, based on the ATC method [32], a feasible way to handle the external variables for the TSO and DSOs is to consider them as parameters. As shown in Fig. 3, with fixed values of the external variables, the coordination within TS and each DS can easily be achieved by separated optimization. The TSO coordinates output of generators and RESs, load pickup and boundary powers $\boldsymbol{P G}_{B}$ in the TS, and the DSO coordinates DG output, load pickup and boundary power $P D_{B}$ in the DS.

The detailed decoupled optimization models for the TSO and DSOs are listed based on (10)-(15).

Upper level model for TSO:

1) Objective function: The objective of the TSO is to maximize (17) to achieve load recovery as much and soon as possible. It contains the amount of TS recovered load, the generator output amount and the penalty function of a load restoration strategy. Since the generator output is proportional to the operation time (18), this objective also provides a suitable 
load pick-up time. $k$ and $l$ are the outer and inner loop numbers which are introduced later.

$$
\begin{aligned}
\operatorname{lil}_{\mathrm{TL}, i}^{k,}, T^{k, l}, p_{\mathrm{RE}, i}^{k, l} P G_{\mathrm{B}, j}^{k, l} & \sum_{i \in N_{\mathrm{TL}}}\left(c_{\mathrm{TL}, i} p_{\mathrm{TL}, i} x_{\mathrm{TL}, i}^{k, l}\right)-\sum_{i \in N_{\mathrm{G}}}\left(p_{\mathrm{G}, i, i n i}+R_{i} T^{k, l}\right) \\
- & \sum_{j \in D i s}\left(v_{j}^{k}\left(P D_{\mathrm{B}, j}^{k, l *}-P G_{\mathrm{B}, j}^{k, l}\right)+\left\|w_{j}^{k} \circ\left(P D_{\mathrm{B}, j}^{k, l *}-P G_{\mathrm{B}, j}^{k, l}\right)\right\|_{2}^{2}\right)
\end{aligned}
$$

2) Power supply constraints: The power supply in TS contains different types of traditional generators such as hydro unit, steam unit and combustion turbines, and RESs such as large-scale wind farms. In the load restoration stage, the conventional generators are ramping up with ramp rate $r_{i}$ between the upper and lower limits [30] and the related constraints are presented in (18). Restoration step time is shown in (19) and wind farms output limitation is (20).

$$
\begin{gathered}
p_{\mathrm{G}, i}=\max \left\{p_{\mathrm{G}, \text { ini }, i}+R_{i} T^{k, l}, p_{\mathrm{G}, i, \min }\right\} \\
\vee \min \left\{p_{\mathrm{G}, \text { ini }, i}+R_{i} T^{k, l}, p_{\mathrm{G}, i, \max }\right\} \forall i \in N_{\mathrm{G}} \\
T_{\min } \leq T^{k, l} \leq T_{\max } \\
p_{\mathrm{RE}, i, \min } \leq p_{\mathrm{RE}, i}^{k, l} \leq p_{\mathrm{RE}, i, \max } \forall i \in N_{\mathrm{RE}}
\end{gathered}
$$

3) Transmission power balance constraints: According to the meshed transmission network, the power balance constraints corresponded to the AC power flow calculation are presented in (21)-(26), where $p_{n}$ and $q_{n}$ are the active and reactive power of node $n, P_{n m}$ and $Q_{n m}$ are the active and reactive power of branch $\mathrm{nm}$.

$$
\begin{gathered}
p_{n}=-x_{\mathrm{TL}, i}^{k, l} p_{\mathrm{TL}, i}\left(i \in N_{\mathrm{TL}}\right) \vee-P G_{\mathrm{B}, j}^{k, l}(j \in D i s) \\
\vee p_{\mathrm{RE}, i}^{k, l}\left(i \in N_{\mathrm{RE}}\right) \vee p_{\mathrm{G}, i}\left(i \in N_{\mathrm{G}}\right) \vee 0 \quad \forall n \in N_{\mathrm{TS}} \\
q_{n}=-x_{\mathrm{TL}, i}^{k, l} q_{\mathrm{TL}, i}\left(i \in N_{\mathrm{TL}}\right) \vee q_{\mathrm{G}, i}^{k, l}\left(i \in N_{\mathrm{G}}\right) \\
\vee q_{\mathrm{B}, i}^{k, l}\left(i \in N_{\mathrm{B}}\right) \vee 0 \quad \forall n \in N_{\mathrm{TS}} \\
p_{n}=\sum_{\forall n, m \in N_{\mathrm{TS}}, n \neq m} P_{n m} \\
q_{n}=\sum_{\forall n, m \in N_{\mathrm{TS}}, n \neq m} Q_{n m} \\
P_{n m}=V_{n}^{2} g_{n m}-V_{n} V_{m}\left(g_{n m} \cos \theta_{n m}+b_{n m} \sin \theta_{n m}\right) \forall n, m \in N_{\mathrm{TS}} \\
Q_{n m}=-V_{n}^{2} b_{n m}-V_{n} V_{m}\left(g_{n m} \sin \theta_{n m}-b_{n m} \cos \theta_{n m}\right) \forall n, m \in N_{\mathrm{TS}}
\end{gathered}
$$

4) Security constraints: As security constraints, branch flow, voltage, reserve constraints and boundary power limitation are (27), (28), (29)-(30) and (31), respectively. Therein, constraint (18) is a linear frequency limit considering the total capacity of restored generators $s$ and their frequency response constant $\varepsilon$ [33]. Specially, considering the $N-1$ security criterion of generator, (29) and (30) ensure that the required spinning reserve and dynamic reserve are provided in the restoration procedure. Equation (31) is the boundary power flow limit between the TS and each DS, and its number equals to the number of distribution systems.

$$
\begin{array}{rr}
S_{n m}^{2}=P_{n m}^{2}+Q_{n m}^{2} \leq S_{n m, \text { max }}^{2} & \forall n \in N_{\mathrm{TS}} \\
V_{n, \text { min }}<V_{n}<V_{n, \max } & \forall n \in N_{\mathrm{TS}} \\
\Delta f=\left|\sum_{i \in N_{\mathrm{TL}}} x_{\mathrm{TL}, i}^{k, l} p_{\mathrm{TL}, i}+\sum_{j \in D i s} P G_{\mathrm{B}, j}^{k, l}-\sum_{i \in N_{\mathrm{RE}}} p_{\mathrm{RE}, i}^{k, l}\right| / \sum_{i^{e} \in N_{\mathrm{G}}, i^{e} \neq i} \frac{S_{i^{e}}}{\varepsilon_{i}} \mid \leq \Delta f_{\mathrm{max}}
\end{array}
$$

$$
\begin{aligned}
p_{\mathrm{G}, i} \leq & \sum_{i^{e} \in N_{\mathrm{G}}, i^{e} \neq i}\left(p_{\mathrm{G}, i^{e}, \text { max }}-p_{\mathrm{G}, i^{e}, \text { min }}+p_{\mathrm{G}, i^{e}}^{k, l}\right) \\
& -\boldsymbol{x}_{\mathrm{TL}}^{k, l} \boldsymbol{p}_{\mathrm{TL}}^{\mathrm{T}}-\sum_{j \in D i s} P G_{\mathrm{B}, j}^{k, l}+\sum_{i \in N_{\mathrm{RE}}} p_{\mathrm{RE}, i}^{k, l} \quad \forall i \in N_{\mathrm{G}} \\
- & P_{\mathrm{B}, j, \max } \leq P G_{\mathrm{B}, j}^{k, l} \leq P_{\mathrm{B}, j, \max } \quad \forall j \in D i s
\end{aligned}
$$

Note that the operation conditions of DSs are considered in the TS restoration optimization model through the target variables $P D_{\mathrm{B}}^{*}$.

Lower level model for DSO $j$ :

1) Objective function: The objective of the DSO is to maximize the load pickup amount in its control area considering the penalty function. $N_{\mathrm{DL}, j}, N_{\mathrm{DG}, j}$ and $N_{\mathrm{DS}, j}$ are sets of load, DG and node in $j$ th DS, respectively.

$$
\begin{gathered}
\operatorname{Max}_{x_{\mathrm{DL}, j, i}^{k,}, P D_{\mathrm{B}, j}^{k, l}, p_{\mathrm{DG}, i}} \sum_{i \in N_{\mathrm{DL}, j}}\left(c_{\mathrm{DL}, j, i} p_{\mathrm{DL}, j, i} x_{\mathrm{DL}, j, i}^{k, l}\right)-v_{j}^{k}\left(P D_{\mathrm{B}, j}^{k, l}-P G_{\mathrm{B}, j}^{k, l^{*}}\right) \\
-\left\|w_{j}^{k} \circ\left(P D_{\mathrm{B}, j}^{k, l}-P G_{\mathrm{B}, j}^{k, l^{*}}\right)\right\|_{2}^{2}
\end{gathered}
$$

2) Power supply constraints: The power supply in the DS is DGs, and the related DG output constraints are (22)-(23).

$$
\begin{gathered}
p_{\mathrm{DG}, i, \text { min }} \leq p_{\mathrm{DG}, i} \leq p_{\mathrm{DG}, i, \max } \forall i \in N_{\mathrm{DG}, j} \\
q_{\mathrm{DG}, i, \text { min }} \leq q_{\mathrm{DG}, i} \leq q_{\mathrm{DG}, i, \max } \forall i \in N_{\mathrm{DG}, j}
\end{gathered}
$$

3) Distribution power balance constraints: Equations (35)-(36) are DisFlow equations [17] a unidirectional single branch radial distribution network. $P_{\mathrm{DS}, n}$ and $Q_{\mathrm{DS}, n}$ are active and reactive power flow from node $n$ in the DS branch.

$$
\begin{gathered}
p_{\mathrm{DS}, n}=-x_{\mathrm{DL}, j, i}^{k, l} p_{\mathrm{DL}, i}\left(i \in N_{\mathrm{DL}, j}\right) \vee p_{\mathrm{DG}, i}\left(i \in N_{\mathrm{DG}, j}\right) \\
\vee P D_{\mathrm{B}, j}^{k, l}(j \in D i s) \vee 0 \quad \forall n \in N_{\mathrm{DS}, j} \\
q_{\mathrm{DS}, n}=-x_{\mathrm{DL}, j, i}^{k, l} q_{\mathrm{DL}, i}\left(i \in N_{\mathrm{DL}, j}\right) \vee q_{\mathrm{DG}, i}\left(i \in N_{\mathrm{DG}, j}\right) \\
\vee q_{\mathrm{B}, j}^{k, l}(j \in D i s) \vee 0 \quad \forall n \in N_{\mathrm{DS}, j} \\
P_{\mathrm{DS}, n_{\text {in }}}=P_{\mathrm{DS}, n_{\text {out }}}-r_{n_{\text {out }}} \frac{P_{\mathrm{DS}, n_{\text {out }}}^{2}+Q_{\mathrm{DS}, n_{\text {out }}}^{2}}{V_{n_{\text {out }}}^{2}}-p_{\mathrm{DS}, n_{\text {in }}} \\
\forall n_{\text {in }} \in N_{\text {in }, j}, n_{\text {out }} \in N_{\text {out }, j} \\
Q_{\mathrm{DS}, n_{\text {in }}}=Q_{\mathrm{DS}, n_{\text {out }}}-x_{n_{\text {out }}} \frac{P_{\mathrm{DS}, n_{\text {out }}}+Q_{\mathrm{DS}, n_{\text {out }}}^{2}}{V_{n_{\text {out }}}^{2}}-q_{\mathrm{DS}, n_{\text {in }}} \\
\forall n_{\text {in }} \in N_{\text {in }, j}, n_{\text {out }} \in N_{\text {out }, j}
\end{gathered}
$$

4) Security constraints: Security constrains in DS are voltage limit (39)-(40), power loss limit (41) and boundary power limit (42). This model assumes that the switch devices are available in the distribution network, hence, loads can be connected/disconnected [34], [36]-[37].

$$
\begin{aligned}
& V_{n_{\text {out }}}^{2}=V_{n_{\text {in }}}^{2}-2\left(r_{n_{\text {in }}} P_{\mathrm{DS}, n_{\text {in }}}+x_{n_{\text {in }}} Q_{\mathrm{DS}, n_{\text {in }}}\right) \\
& +\left(r_{n_{\mathrm{in}}}^{2}+x_{n_{\mathrm{in}}}^{2}\right) \frac{P_{\mathrm{DS}, n_{\mathrm{in}}}^{2}+Q_{\mathrm{DS}, n_{\mathrm{in}}}^{2}}{V_{n_{\mathrm{in}}}^{2}} \forall n_{\mathrm{in}} \in N_{\mathrm{in}, j}, n_{\text {out }} \in N_{\text {out }, j} \\
& V_{n, \text { min }}<V_{n}<V_{n, \text { max }} \forall n \in N_{\mathrm{DS}, j} \\
& L_{n}=r_{n} \frac{P_{\mathrm{DS}, n}^{2}+Q_{\mathrm{DS}, n}^{2}}{V_{n}^{2}} \leq L_{n, \max } \forall n \in N_{\mathrm{DS}, j} \\
& -P_{\mathrm{B}, j, \max } \leq P D_{\mathrm{B}, j}^{k, l} \leq P_{\mathrm{B}, j, \max } \quad j \in \text { Dis }
\end{aligned}
$$


In the $j$ th DS optimization model, the TS condition is considered by embedding the response variables $P G_{\mathrm{B}, j}^{*}$.

\section{B. Iterative Interaction Between TSO and DSO}

Through decoupled optimization, the TSO coordinates the entities in the TS and the DSO coordinates the ones in the DS. However, the coordination between the TS and DSs is required to complete the decentralized decision-making. In order to address this problem, an inner-outer iteration is proposed based on the ATC method [31]-[32].

Fig. 4 shows the iterative interaction process. The inner loop is the interaction between TSO and DSO and the outer loop updates penalty multipliers of model decoupling. In each iteration, the TSO and DSOs independently determine their restoration strategies based on the TSO Model (17)-(31) or DSO Model (32)-(42). Then, the values of boundary power are sent to the other level. DSOs acquire the value of response variable $P D_{\mathrm{B}}$ from the TSO. Then, as the result of the decentralized decision-making, DSOs sent back the value of the target variable $P G_{\mathrm{B}}$ to the TSO. On the contrary, the TSO receives the target variables $P G_{\mathrm{B}}$ with fixed values for decisionmaking and provides the values of response variables $P D_{\mathrm{B}}$ to DSOs. The detailed inner-outer loop calculation for the iterative interaction is shown in Appendix A.

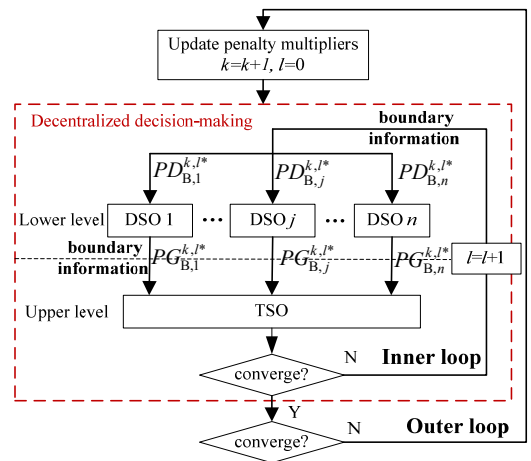

Fig. 4. ATC based iterative process of TS-DS interaction

The TS-DS interaction is the boundary information exchange between the TS and DS. The boundary information refers to the value of boundary power calculated by the TSO and each DSO, respectively. Thus, although there is a bulk system and complex operation information in the coordinated restoration of TS-DS system, the decentralized decisionmaking is implemented by interaction of boundary power. The restoration strategies from different subsystems influence each other by the coordination of boundary power between the TS and DS. Even though the TSO and DSOs have different objectives, the overall objective of the TS-DS system is still realized through the coordination of the decentralized decisionmaking.

The combination of updating the Lagrangian multiplier and augmented Lagrangian penalty function is the method of multipliers, which is proven to converge to the optimal solution of the original problem when models are convex [37]. That means although decisions are made in a decentralized way, the overall system performance target can still be achieved. The ATC method with the method of multipliers makes the decen- tralized decision-making framework possible to implement, however, its convergence requires convexity of the objective function and constraints [38] while the models of the TSO and DSOs are non-convex and discontinuous with complex constraints and tremendous binary variables of load pick-up. Since the quadratic term of the penalty function with a large value of $\boldsymbol{w}$ can act as a local "convexifier", the ATC method may provide a result for the non-convex problem. However, this will lead to a compromise for the global optimality with an unacceptable optimization gap for the load restoration models. These problems are addressed in Section IV.

\section{Decentralized Optimization Method to Solve Non- CONVEX RESTORATION PROBLEM}

In this section, the TL_ATC method is proposed to guarantee the convergence of decentralized optimization of the nonconvex restoration model. First, the original models of the TSO and DSO are transformed into mixed-integer quadratic programming (MIQP) and MILP models. Using these preprocessed models, a three loop iterative algorithm is designed to obtain the optimal decentralized restoration strategy. Applying the TL_ATC method, the results of the MIQP models can be obtained by iteratively performing QP and MILP.

\section{A. Pre-processing of TSO and DSO Optimization Models}

The pre-processing, which includes constraints linearization and binary variable relaxation, is to construct different mathematical forms of the TSO and DSO optimization models. For the TSO model, a linear-programming approximation of AC power flow (LPAC) method [39] is employed to achieve linearization of power flow constraints. Then, (25)-(26) can be replaced by (43)-(47). Besides, the quadratic constraint of branch flow (27) is linearized by (48)-(51).

$$
\begin{array}{r}
\tilde{V}_{n}=1+\delta_{n} \quad \delta_{n} \text { (p.u.) } \in\left(-V_{n},+\infty\right) \\
P_{n m}=g_{n m}-g_{n m} \cos ^{*} \theta_{n m}-b_{n m}\left(\theta_{n}-\theta_{m}\right) \\
Q_{n m}=-b_{n m}-g_{n m}\left(\theta_{n}-\theta_{m}\right)+b_{n m} \cos ^{*} \theta_{n m}-b_{n m}\left(\delta_{n}-\delta_{m}\right) \\
\cos ^{*} \theta_{n m} \geq \cos \theta_{\Delta}^{0} \\
\cos ^{*} \theta_{n m} \leq-\sin \left(v d-\theta_{\Delta}^{0}\right)\left(\theta_{n}-\theta_{m}-v d+\theta_{\Delta}^{0}\right)+\cos \left(v d-\theta_{\Delta}^{0}\right) \\
v=1,2, \ldots, 2 h \\
-S_{n m, \text { max }} \leq P_{n m} \leq S_{n m, \text { max }} \\
-S_{n m, \text { max }} \leq Q_{n m} \leq S_{n m, \text { max }} \\
-\sqrt{2} S_{n m, \text { max }} \leq P_{n m}+Q_{n m} \leq \sqrt{2} S_{n m, \text { max }} \\
-\sqrt{2} S_{n m, \text { max }} \leq P_{n m}-Q_{n m} \leq \sqrt{2} S_{n m, \text { max }}
\end{array}
$$

For the DSO model, the linearized DisFlow model in [17] is used. Then, (37)-(39) and (41) are respectively transformed into (52)-(54) and (55)-(58). The details of this linearization method are given in [17]. Note that $n$ means the branch $n$.

$$
\begin{aligned}
& P_{\mathrm{DS}, n_{\mathrm{in}}}=P_{\mathrm{DS}, n_{\text {out }}}-p_{\mathrm{DS}, n_{\mathrm{in}}} \\
& Q_{\mathrm{DS}, n_{\text {in }}}=Q_{\mathrm{DS}, n_{\text {out }}}-q_{\mathrm{DS}, n_{\mathrm{in}}} \\
& V_{n_{\text {in }}}=V_{n_{\text {out }}}-\frac{r_{n_{\text {out }}} P_{\mathrm{DS}, n_{\text {out }}}+x_{n_{\text {out }}} Q_{\mathrm{DS}, n_{\text {out }}}}{V_{0}}
\end{aligned}
$$




$$
\begin{array}{r}
-V_{0} \sqrt{L_{n, \text { max }} / r_{n}} \leq P_{\mathrm{DS}, n} \leq V_{0} \sqrt{L_{n, \text { max }} / r_{n}} \\
-V_{0} \sqrt{L_{n, \text { max }} / r_{n}} \leq Q_{\mathrm{DS}, n} \leq V_{0} \sqrt{L_{n, \text { max }} / r_{n}} \\
-V_{0} \sqrt{2 L_{n, \text { max }} / r_{n}} \leq P_{\mathrm{DS}, n}+Q_{\mathrm{DS}, n} \leq V_{0} \sqrt{2 L_{n, \text { max }} / r_{n}} \\
-V_{0} \sqrt{2 L_{n, \text { max }} / r_{n}} \leq P_{\mathrm{DS}, n}-Q_{\mathrm{DS}, n} \leq V_{0} \sqrt{2 L_{n, \text { max }} / r_{n}}
\end{array}
$$

Up to now, the TSO Model M1 in (59) and the DSO Model M2 in (60) are MIQP models with binary variable vectors $\boldsymbol{x}_{\mathrm{TL}}$ and $\boldsymbol{x}_{\mathrm{DL}}$. If $\boldsymbol{x}_{\mathrm{TL}}$ and $\boldsymbol{x}_{\mathrm{DL}}$ are relaxed to continuous ones, M1 and $\mathrm{M} 2$ are transformed to quadratic programming (QP) models.

$$
\begin{aligned}
& \mathrm{M} 1 \text { : objective }(17) \\
& \quad \text { s.t. }(18)-(24),(28)-(31),(43)-(51) \\
& \mathrm{M} 2 \text { : objective }(32) \\
& \quad \text { s.t. }(33)-(36),(40),(42),(52)-(58)
\end{aligned}
$$

In addition, the completely independent models of the TSO (M3) and DSO (M4) are created to perform the TL_ATC method. Both M3 in (46) and M4 in (47) are MILP models. $z$ is the iterative index of the TL_ATC method.

$$
\begin{aligned}
\mathrm{M} 3: & \operatorname{Max} \sum_{i \in N_{\mathrm{TL}}}\left(c_{\mathrm{TL}, i} p_{\mathrm{TL}, i} x_{\mathrm{TL}, i}^{z}\right)-\sum_{i \in N_{\mathrm{G}}}\left(p_{G, i, i n i}+R T^{z}\right) \\
\text { s.t. } & (18)-(24),(28)-(31),(43)-(51) \\
\mathrm{M} 4: \operatorname{Max} & \sum_{i \in N_{\mathrm{DL}, j}}\left(c_{\mathrm{DL}, j, i} p_{\mathrm{DL}, j, i} x_{\mathrm{DL}, j, i}^{z}\right) \\
\text { s.t. } & (33)-(36),(40),(42),(52)-(58)
\end{aligned}
$$

\section{B. Iteration Process of TL_ATC}

Based on the inner-outer loop iteration of the ATC method, the third loop with the iterative index $z$ is added to form the three loop iterative structure. The third loop is designed to deal with the binary variables in the restoration model. Three types of models are required in the proposed TL_ATL method as shown in Fig. 5: Type1: M1 and M2 with the binary variables being set as continuous variables between 0-1, Type2: M1 and M2 with binary variables the fixed values of load pickup and Type3: M3 and M4 with boundary power with fixed values. The final restoration strategy of the TS-DS system can be determined as long as the convergence conditions of the three loops iteration are satisfied.

Using the Models in (59)-(62) (M1, M2, M3 and M4), the flowchart of the TL_ATC method is shown in Fig. 5, and the detailed iterative process is listed as follows:

Step 0: (Initialize) Set $z=0$ and obtain TSO and DSO models M1, M2, M3 and M4.

Step 1: (Solve the QP problem) Apply the ATC method to M1 as the upper level model and M2 as the lower level models. Note that load pickup variables in $\boldsymbol{x}_{\mathrm{TL}}$ and $\boldsymbol{x}_{\mathrm{DL}}$ are all relaxed to continuous variables. Therefore, models in this stage are quadratic programming $(\mathrm{QP})$ problems.

Step 2: (Solve the MILP problem) Use the optimal boundary power $\boldsymbol{P}_{\mathrm{B}}^{z}$ obtained from Step 1 . The MILP models M3 and M4 are solved with fixed $P D_{\mathrm{B}}^{k, l}$ and $P G_{\mathrm{B}}^{k, l}$, respectively. Note that load pickup variables in $\boldsymbol{x}_{\mathrm{TL}}$ and $\boldsymbol{x}_{\mathrm{DL}}$ are binary variables and the optimal result is obtained by solving the MILP model.

Step 3: (Check convergence) Check (63)-(65) where $F$ is the summary value of TSO objective (17) and DSO objectives (32). If (63) and (64) are satisfied or the iteration limit (65) is reached, the final converged result is obtained, otherwise, proceed to Step 4.

$$
\begin{gathered}
\left|\left(F^{z}-F^{z-1}\right) / F^{z}\right| \leq \varepsilon_{4} \wedge\left|\left(F^{z-1}-F^{z-2}\right) / F^{z-1}\right| \leq \varepsilon_{4} \\
\boldsymbol{x}_{\mathrm{TL}}^{z}=\boldsymbol{x}_{\mathrm{TL}}^{z-1} \wedge \boldsymbol{x}_{\mathrm{DL}, j}^{z}=\boldsymbol{x}_{\mathrm{DL}, j}^{z-1}(\forall j \in D i s) \\
z \geq Z
\end{gathered}
$$

Step 4: (Solve the QP problem) Set $z=z+1$. Applying ATC to M1 and M2 with fixed load pickup variables obtained from Step 2. Since the binary variables are fixed, the model is a QP problem.

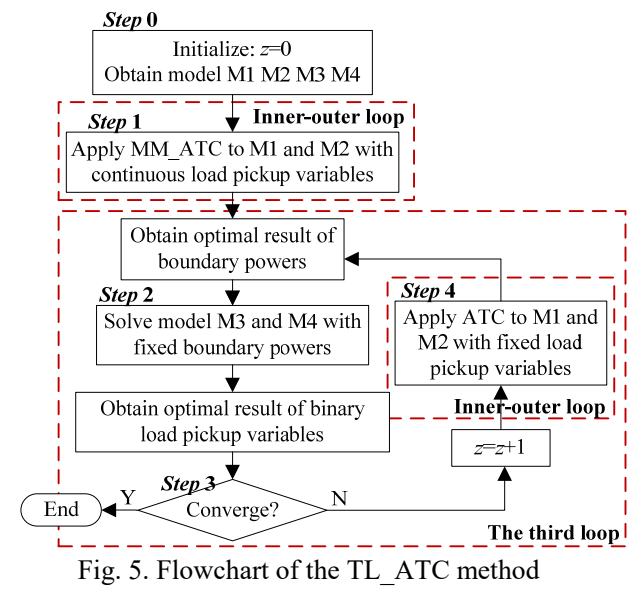

As shown in Fig. 5, the TL_ATC method still has the form of decentralized optimization. The TL_ATC method is feasible as long as the models of the TSO and DSO are convex after relaxing the binary variables. Consequently, it is convenient to integrate the existing TS restoration models and the general operation models of DS. The convergence of the TL_ATC is proved in Appendix B.

The TL_ATC method improves the ATC method by computational scale reduction and mathematical programming model simplification. Compared with the ATC method, the TL_ATC method reduces computational scale of the models by decreasing the number of variables and related constraints. Besides, the TL_ATC method performs QP and MILP, while the ATC method does MIQP. Overall, the TL_ATC method not only guarantees the convergence but also improves the computation efficiency by solving the models with the simpler mathematical programming form and smaller computational scale.

\section{CASE Study}

Three systems are used to demonstrate the effectiveness of the proposed method. The first one, named as T6D2, including one six-bus TS and two ADSs. The second system is T118D30 which contains the IEEE-118-bus test system and 30 ADSs. The third one is a real-world TS-DS system in Dongying City, Shandong province, China. It has 5 major plants in the TS and 23 DGs in DS, 113 nodes, 52 transmission lines and 77 distribution lines of $220 \mathrm{kV}$ and $110 \mathrm{kV}$ voltage level, respectively. All case studies were conducted using Gurobi V7.5.1 on a computer with the Intel(R) Core(TM) i5- 
$2400 \mathrm{CPU}$ and $4 \mathrm{~GB}$ RAM. The initial values of the TS boundary power $P G_{\mathrm{B}}^{1,1}$ for all the tested system are set as 0 . The simulation conditions are presented in Appendix C.

\section{A. Iterative Interaction Process of Determining the Coordinated Restoration Strategy}

The T6D2 system is shown in Fig. 6. There are a TSO for the TS, DSO1 for ADS1 with five loads and two DGs and DSO2 for ADS2 with four loads and two DGs. All the parameters of the T6D2 system are the same as in [11]. The detailed process of obtaining the coordinated restoration strategy is presented as follows.

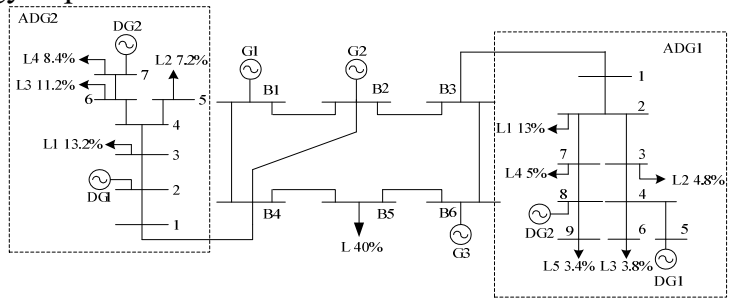

Fig. 6. Six-bus system with two ADGs

i) Firstly, binary variables of load pickup are relaxed to continuous ones to obtain the upper bound of the objective. DSO1 and DSO2 perform decentralized decision-making by parallel optimization calculation. According to the result, the two ADSs determine the target variables, which are the boundary powers of the lower level, as $\boldsymbol{P} \boldsymbol{D}_{\mathrm{B}}=[7.27,15.56]$ MW. Considering the boundary power of DSOs and its own objective, the TSO decides the response variables, which are the boundary powers of the upper level, to be $\boldsymbol{P G}_{\mathrm{B}}=[6.27$, 14.56] MW. After the coordination process through 4 inner loop iterations and 2 outer loop iterations, the values of SVs between the TS and DS are $\boldsymbol{P}_{\mathrm{B}}=[6.65,14.86] \mathrm{MW}$. ii) Secondly, load pickup variables return to binary forms. With fixed SVs in $\boldsymbol{P}_{\mathrm{B}}$, the TSO determines its restoration strategy as $\boldsymbol{x}_{T L}$ $=[1]$ and $\boldsymbol{p}_{\mathrm{G}}=[58.78,36.73,26.27] \mathrm{MW}$, while two DSOs have strategies as $\boldsymbol{p}_{\mathrm{DG}, 1}=[10,12] \mathrm{MW}, \boldsymbol{x}_{\mathrm{DL}, 1}=[1,0,1,0,1]$ and $\boldsymbol{p}_{\mathrm{DG}}$, ${ }_{2}=[20,15] \mathrm{MW}, \boldsymbol{x}_{\mathrm{DL}, 2}=[1,0,1,0]$, respectively. iii) Thirdly, regarding load pickup variables as fixed ones, there is another coordination process for the TS and DSs. Through 2 third loop iterations, the unit generation in the TS is adjusted to be $\boldsymbol{p}_{\mathrm{G}}=[58.15,37.14,26.72] \mathrm{MW}$ and SVs are changed to $\boldsymbol{P}_{\mathrm{B}}=[6$, 16] MW. Applying the new SVs, the load pickup variables do not change. Therefore, the final coordinated restoration strategy of the TS-DS system is obtained.

In the final coordinated restoration strategy, the outputs of G1, G2 and G2 are 58.78 MW, 36.73 MW and 26.27 MW, respectively, and the outputs of DGs in the DS1 are $10 \mathrm{MW}$ and $12 \mathrm{MW}$ while the outputs of DGs in the DS2 are $20 \mathrm{MW}$ and $15 \mathrm{MW}$. Set the initial time of the TS-DS coordination as 0 min, then, at $8.89 \mathrm{~min}$, load L of the TS, L1 L3 L4 of DS1 and L1 L3of DS2 are picked up. Applying this strategy, the boundary exchange powers are $6 \mathrm{MW}$ for TS-DS1 and 16 MW for the TS-DS2.

\section{B. Performance of TL ATC using the T118D30 system}

T118D30 system has 118-bus transmission system with 30 ADSs. The ADS is the IEEE-33 DS with five DGs. The computation performance of this case is listed in Table I. Set $\beta=1$. The iteration limitation of the inner, outer and the third loop for the T118D30 system are all 50.

For the purpose of comparison, the original ATC method is also applied in the system. The calculation time, objective value $F$ and iteration number under different convergence thresholds are shown in the Table I. Besides, the objective value of the centralized optimization is demonstrated to show the optimization gap of these two methods. According to the result, a higher requirement of convergence thresholds increases iteration numbers of the ATC and TL_ATC, however, the latter performs better in computation time. That is because the mathematical programming models in the TL_ATC method are QP and MILP while the ATC method performs MIQP. Based on the TL_ATC method, model optimizations in the iteration process are with many fixed variables so that the computational scales are smaller.

TABLE I

COMPUTATION PERFORMANCE OF DIFFERENT METHODS

\begin{tabular}{|c|c|c|c|c|c|c|c|}
\hline SYS & & ATC1 & ATC2 & ATC3 & TL1_ATC & TL2_ATC & TL3_ATC \\
\hline \multirow{6}{*}{$\begin{array}{l}\text { T118 } \\
\text { D30 }\end{array}$} & $\begin{array}{c}\text { calculation } \\
\text { time }(\mathrm{s})\end{array}$ & 11.55 & / & / & 8.53 & 9.47 & 11.32 \\
\hline & $F$ & -2740.91 & 1 & 1 & -2714.32 & -2714.15 & -2712.83 \\
\hline & $\begin{array}{c}\text { Optimization } \\
\text { Gap }\end{array}$ & $1.15 \%$ & l & / & $0.18 \%$ & $0.71 \%$ & $0.12 \%$ \\
\hline & $\begin{array}{c}\text { Iteration } \\
(k, l) /(z, k, l)\end{array}$ & $(2,12)$ & / & / & $(3,8,21)$ & $(3,8,24)$ & $(4,11,32)$ \\
\hline & $\begin{array}{c}\text { Convergence } \\
\text { threshold }\end{array}$ & $\begin{array}{l}\varepsilon_{1}=0.1 \\
\varepsilon_{2}=0.1 \\
\varepsilon_{3}=0.1\end{array}$ & $\begin{array}{l}\varepsilon_{1}=0.01 \\
\varepsilon_{2}=0.01 \\
\varepsilon_{3}=0.01\end{array}$ & $\begin{array}{c}\varepsilon_{1}=0.01 \\
\varepsilon_{2}=0.001 \\
\varepsilon_{3}=0.01\end{array}$ & $\begin{array}{l}\varepsilon_{1}=0.1 \\
\varepsilon_{2}=0.1 \\
\varepsilon_{3}=0.1 \\
\varepsilon_{4}=0.1\end{array}$ & $\begin{array}{l}\varepsilon_{1}=0.01 \\
\varepsilon_{2}=0.01 \\
\varepsilon_{3}=0.01 \\
\varepsilon_{4}=0.01\end{array}$ & $\begin{array}{l}\varepsilon_{1}=0.01 \\
\varepsilon_{2}=0.001 \\
\varepsilon_{3}=0.01 \\
\varepsilon_{4}=0.01\end{array}$ \\
\hline & Centralized & \\
\hline
\end{tabular}
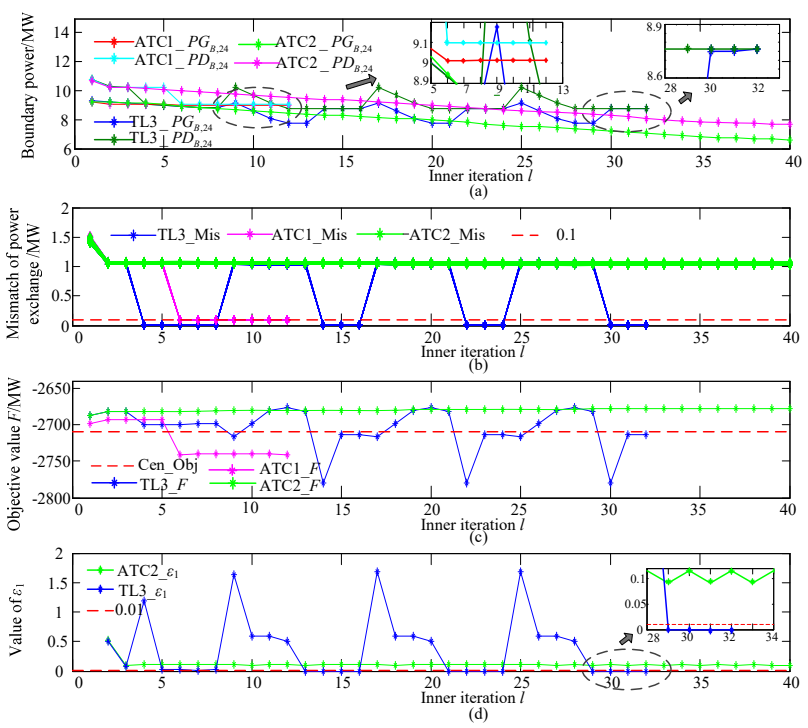

Fig. 7. Target and response power of bus 24 (a), boundary power mismatch between TS and DS of 30 buses (b), objective value changes (c) in iteration process and the status of convergence threshold (d).

For the T118D30 system, the convergence problem of the ATC method appears when the convergence thresholds are set as 0.01 . According to Fig. 7 , both target variable $P D_{\mathrm{B}}$ and response variable $P G_{\mathrm{B}}$ try to be close to each other in the iteration process with the ATC or TL_ATC method. However, the gap is difficult to be eliminated with the ATC method as shown by ATC2_PD $D_{\mathrm{B}, 24}$ and ATC2_PG $G_{\mathrm{B}, 24}$ in Fig. 7 (a). The mismatch of boundary power between the TS and DS cannot reach the required convergence thresholds 0.01 by applying the ATC method, while the TL_ATC making the mismatch 
TL3_Mis less than 0.001 MW (Fig. 7 (b)). The mismatch ATC1_Mis equals to almost $1.1 \%$, and much higher than the acceptable $0.1 \%$ mismatch in the decentralized computation method. Moreover, the multi-step restoration process will accumulate the error, which may finally lead to security violation. Thus, the ATC is not feasible in this case. In Fig. 7 (c), the changes of the objective function values of ATC $\left(\mathrm{ATC} 1 \_/ \mathrm{ATC} 2 \_F\right)$ and TL_ATC $\left(\mathrm{TL} 3 \_F\right)$ in the iteration process are presented. The objective function value of the TL_ATC is higher than ATC and close enough to the centralized one with $0.12 \%$ optimization gap. Fig. 7 also shows the iteration process of the ATC when the required convergence thresholds are 0.01 (ATC2). In this condition, the reason of the non-convergence problem is the convergence threshold ATC2 $\varepsilon_{1}$ cannot reach 0.01 as shown in Fig. 7 (d). This further leads to the target variable $P D_{\mathrm{B}}$ and response variable $P G_{\mathrm{B}}$ not close to each other by the outer loop in (a) and the mismatch of each boundary power ATC2_Mis is beyond 1 MW in (b). Therefore, the result is infeasible although the objective values ATC2 $F$ is better than the centralized optimization. In summary, the TL_ATC method not only has better convergence performance but also improves the restoration strategy by a $28.08 \mathrm{MW}$ increase of the objective function value.

\section{Comparison of the Conventional Restoration Strategy and Coordinated One in Real-world System}

In the conventional restoration strategy of city Dongying, the load side includes the loads connected to the TS directly and DSs with or without DGs. The DSs participate in the process as load blocks with fixed load amount. Considering the integration of DGs, the operation center of city Dongying would like to take advantage of the 'active' characteristic of load side to benefit bulk system restoration. Thus, a coordinated restoration strategy is provided by applying the proposed decentralized restoration scheme. For comparison, the conventional restoration scheme, which separates the TS and DSs and regards DSs as load blocks with fixed load amount, is also applied. An optimistic assumption is made in the conventional scheme: DGs have the largest output, thus, each DS keeps the possible lowest total load amount.

TABLE II

RESTORATION EFFICIENCY COMPARISON OF DECENTRALIZED COORDINATION SCHEME AND CONVERSIONAL SEPARATED SCHEME

\begin{tabular}{cc|ccccccc}
\hline \hline \multicolumn{2}{c}{ Steps } & S1 & S2 & S3 & S4 & S5 & S6 & S7 \\
\hline \multirow{2}{*}{ Decen } & Cal_t (s) & 6.83 & 4.71 & 3.85 & 13.31 & 7.35 & 2.26 & 4.47 \\
\cline { 2 - 8 } & OP_gap & $0.10 \%$ & $0.46 \%$ & $0.11 \%$ & $0.08 \%$ & $0.34 \%$ & $0.17 \%$ & $0.26 \%$ \\
\hline \multirow{2}{*}{$\begin{array}{c}\text { Objective- Decen } \\
\text { values }\end{array}$} & -38.14 & 122.45 & 151.62 & 275.64 & 265.12 & 259.61 & 284.03 \\
\cline { 2 - 8 } & Conve & -229.66 & -47.66 & 27.34 & 27.34 & 27.34 & 27.34 & 27.34 \\
\hline \multirow{2}{*}{$\begin{array}{c}\text { Load } \\
\text { recovery }\end{array}$} & Decen & $10.15 \%$ & $31.57 \%$ & $43.54 \%$ & $62.57 \%$ & $74.38 \%$ & $87.74 \%$ & $100 \%$ \\
\cline { 2 - 8 } & Conve & $6.80 \%$ & $23.84 \%$ & $38.14 \%$ & $51.95 \%$ & $63.21 \%$ & $71.83 \%$ & $83.28 \%$ \\
\hline \hline
\end{tabular}

The restoration efficiency comparison between the decentralized coordination scheme (Decen) and the conventional separated scheme (Conve) is shown in Table II. As can be seen, with the computation time in seconds $(\mathrm{Cal} \mathrm{t})$ and the optimization gap within $0.5 \%$ (OP_gap), the proposed TL_ATC method can provide the coordination restoration scheme with high computation efficiency. According to Table II, the coordination scheme improves the objective values in each step and speeds up the load recovery process. Fig. 8 shows the load restoration conditions in detail. Even though a positive assumption (DGs have the largest output) is made in the conventional restoration method, the coordinated one still has better performance. The load recovery (L1) of the coordinated restoration is completed in 7 steps with $67.17 \mathrm{~min}$, while the conventional one (L2) takes 9 steps with $82.14 \mathrm{~min}$.

In the step-by-step restoration process, the voltage and frequency will reach the worst conditions if all the selected loads in the strategy of the current step are picked up at the beginning of this time step. The security violation problem can be avoided if the worst security conditions are within limitations. Fig. 9 shows the worst security condition in different timeinterval according to the restoration strategies. Taking bus 3 of the TS as an example, the voltage status of each step in the TS with coordinated strategy (TV3) and conventional strategy (CTV3) as well as Buses 3 and 8 in DS3 (DV3_3 and DV3_8) are shown in Fig. 9 (a). Fig. 9 (b) demonstrates the coordinated and conventional frequency deviations (Coo_f and Con_f) in each step. Accordingly, the voltage and frequency of the two schemes both can be kept within the security constraints (the red dotted line) even in the worst condition. That means the coordination method speeds up the restoration process with the same security constraints as the conventional one. The better performance depends on the efficient matching of power supply and demand by the coordination in the TS-DS system, which is further explained in Fig. 10.

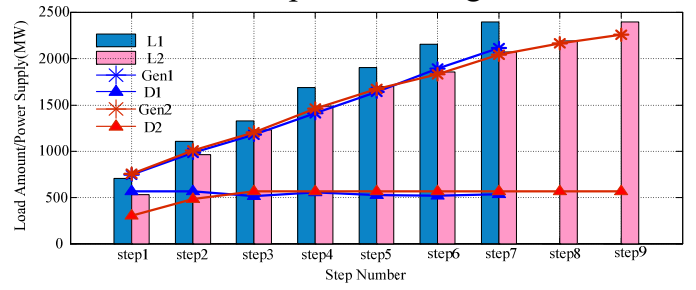

Fig. 8. Load recovery, generators and DGs generation in multi-step
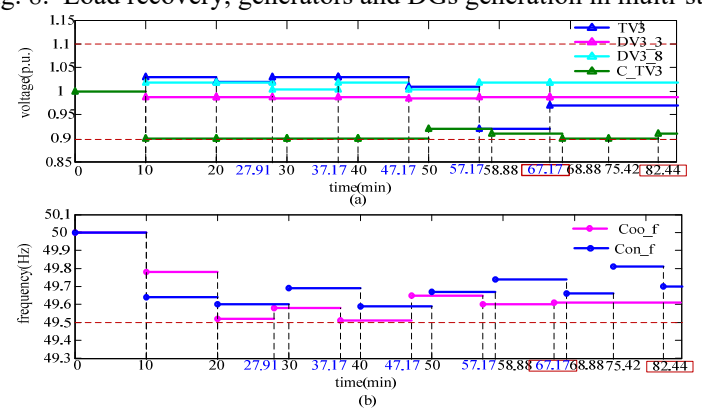

Fig. 9. Voltages of TS and DS (b) and frequencies (c) of coordinated restoration and conventional restoration in the worst condition of each time interval

There are totally 346 iterations in 7 steps to achieve the whole coordinated restoration process. Buses 3 and 6 are chosen to be examples in Fig. 10. The goal of iteration in each step is to fulfill the consistency of the TS boundary power $(P G 3 / P G 6)$ and the corresponding DS boundary power $(P D 3 / P D 6)$. SR means the finally result of each step with inner, outer and the third loop number. The efficient coordination of sources and loads has two aspects. One aspect is the flexible adjustment of DGs in the DS. As can be seen in Fig. 10 , the DG output of ADS3 in each step is different from each 
other. This part of power supply can be adjusted flexibly according to the restoration requirement of each step and improves the objective function value. The other aspect is the open choice of the role, a power consumer or a power supplier, that the load side plays. In Fig. 10, the value of boundary power of Bus 6 is negative in the former steps. That means the coordinated restoration allows the load side to provide TS with power support when necessary. This action benefits the restoration by improving the whole objective function value and restoring more important loads in the earlier stage.

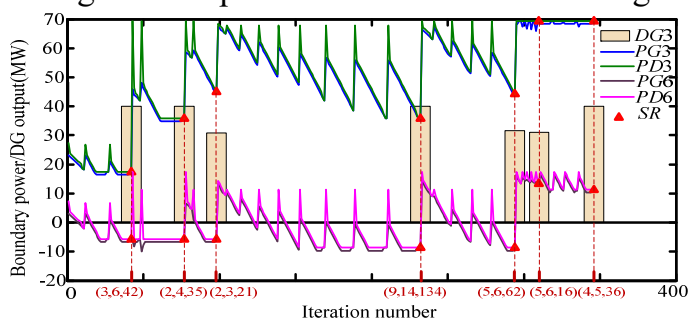

Fig. 10. Boundary powers of bus 3 and bus 6 of TS in the iteration process of coordinated restoration

\section{CONCLUSION}

A new coordinated restoration of the TS and DSs is presented considering the operation of DSs in the bulk system restoratio. A decentralized restoration scheme, which contains the decentralized decision-making framework and the TL_ATC decentralized optimization method, is designed to achieve the coordinated restoration. With the decentralized decision-making framework, the coupled TS-DS restoration system can be coordinated by independent optimization of subsystems and simple information interaction between TSO and DSOs. The TL_ATC method provides reliable decentralized restoration strategy by guaranteeing the convergence of decentralized optimization with non-convex models. The case study demonstrates the detailed coordinated restoration process and the superiority of the TL_ATC method. By applying the proposed method to a real-world system, the coordinated restoration is proved to be more efficient to enhance resilience than the conventional one.

Concentrating on the spatial coordination in the restoration process of the TS-DS system, this paper constructs the decentralized restoration scheme. To achieve the spatial-temporal coordination of the TS-DS restoration, further work will focus on the dynamic process of restoration as well as the uncertainty of RESs.

\section{APPENDIX A}

The ATC based inner-outer loop calculation under the decentralized decision-making framework.

Step 0: (Initialize) Set $k=0$ for the outer loop and $l=0$ for the inner loop. Define the values of $P G_{\mathrm{B}, j}^{k, l}, v_{j}^{k}$ and $w_{j}^{k}(j \in D i s)$ for the first iteration.

Step 1: (Inner loop) Set $l=l+1.1$ ) DSO: Solve model (18)(27) with fixed $P G_{\mathrm{B}, j}^{k, * *}$ from the last iteration and get $P D_{\mathrm{B}, j}^{k l}$. 2) TSO: Solve model with fixed $P D_{\mathrm{B}_{i}}^{k, l^{*}}(6)-(17)$ and obtain $P G_{\mathrm{B}, j}^{k, l+1}$ for next iteration in the DSO part.

Step 2: (Check convergence) 1) Inner loop check: Check the inner loop convergence equations (A-1), (A-2). If they are satisfied or the inner iteration limit (A-3) is reached, proceed to 2) for the outer loop check, otherwise, return to step 1.

$$
\begin{aligned}
& \left|P G_{\mathrm{B}, j}^{k, l}-P G_{\mathrm{B}, j}^{k, l-1}\right| \leq \varepsilon_{1} \quad \forall j \in D i s \\
& \left|P D_{\mathrm{B}, j}^{k, l}-P D_{\mathrm{B}, j}^{k, l-1}\right| \leq \varepsilon_{1} \quad \forall j \in D i s \\
& \quad l \geq L
\end{aligned}
$$

2) Outer loop check: Check equations (A-4), (A-5). $F_{i}$ is the objective of subsystem $i$. If they are satisfied or the outer loop iteration limit (A-6) is reached, the converged solution is obtained, otherwise, go to step 3 .

$$
\begin{gathered}
\left|P G_{\mathrm{B}, j}^{k, l}-P D_{\mathrm{B}, j}^{k, l-1}\right| \leq \varepsilon_{2} \quad \forall j \in \text { Dis } \\
\left|\left(F_{s y s}^{k, l}-F_{s y s}^{k, l-1}\right) / F_{s y s}^{k, l}\right| \leq \varepsilon_{3} \forall s y s \in \mathrm{TS} \vee \mathrm{DSs}(\mathrm{A}-5 \\
k \geq K
\end{gathered}
$$

Step 3: (Outer loop) Set $k=k+1$ and update penalty parameters $v_{j}^{k}$ and $w_{j}^{k}$ according to (A-7) and (A-8).

$$
\begin{aligned}
& v_{j}^{k+1}=v_{j}^{k}+2\left(w_{j}^{k}\right)^{2}\left(P D_{\mathrm{B}, j}^{k, l}-P G_{\mathrm{B}, j}^{k, l}\right) \\
& w_{j}^{k+1}=\beta w_{j}^{k}(\beta \geq 1)
\end{aligned}
$$

\section{APPENDIX B}

The proof of the convergence of the TL_ATC method.

Problem (1) can be rewritten as (B-1).

$$
\begin{array}{ll}
\operatorname{Max} & F\left(\boldsymbol{x}_{\mathrm{L}}, \boldsymbol{p}_{\mathrm{G}}, \boldsymbol{P}_{\mathrm{B}}, \boldsymbol{p}_{\mathrm{DG}}\right) \\
\text { s.t. } & \boldsymbol{x}_{\mathrm{L}} \in \Theta_{1}, \boldsymbol{p}_{\mathrm{G}} \in \Theta_{2}, \boldsymbol{P}_{\mathrm{B}} \in \Theta_{3}, \boldsymbol{p}_{\mathrm{DG}} \in \Theta_{4}
\end{array}
$$

According to the flowchart in Fig. 5, binary variables in $x_{\mathrm{L}}$ are relaxed as continuous variables. Solve the model, then, there is the maximum value of objective $F_{\max }^{*}$ and the optimal result of $\boldsymbol{P}_{\mathrm{B}}$. Note that $F_{\max }^{*}$ can be the upper bound of objective value in model (B-1) with binary variable vector $x_{\mathrm{L}}$.

Define $\boldsymbol{x}_{L}$ as the vector of binary variables. Solve (B-2) with fixed $\boldsymbol{P}_{\mathrm{B}}$ as $\boldsymbol{P}_{\mathrm{B}}^{z}$ and get the optimal result of $\boldsymbol{x}_{\mathrm{L}}, \boldsymbol{p}_{\mathrm{G}}$ and $\boldsymbol{p}_{\mathrm{DG}}$ as $\boldsymbol{x}_{\mathrm{L}}^{z}, \boldsymbol{p}_{\mathrm{G}}^{z}$ and $\boldsymbol{P}_{\mathrm{DG}}^{z}$. Then, formula (B-3) can be obtained.

$$
\begin{gathered}
\operatorname{Max} F\left(\boldsymbol{x}_{\mathrm{L}}, \boldsymbol{p}_{\mathrm{G}}, \boldsymbol{P}_{\mathrm{B}}, \boldsymbol{p}_{\mathrm{DG}}\right) \\
\text { s.t. } \quad \boldsymbol{x}_{\mathrm{L}} \in \Theta_{1}, \boldsymbol{p}_{\mathrm{G}} \in \Theta_{2}, \boldsymbol{P}_{B}=\boldsymbol{P}_{B}^{z}, \boldsymbol{p}_{\mathrm{DG}} \in \Theta_{4} \\
F\left(\boldsymbol{x}_{\mathrm{L}}^{z}, \boldsymbol{p}_{\mathrm{G}}^{z}, \boldsymbol{P}_{\mathrm{B}}^{z}, \boldsymbol{p}_{\mathrm{DG}}^{z}\right) \geq F\left(\boldsymbol{x}_{\mathrm{L}}, \boldsymbol{p}_{\mathrm{G}}, \boldsymbol{P}_{\mathrm{B}}^{z}, \boldsymbol{p}_{\mathrm{DG}}\right) \\
\forall \boldsymbol{x}_{\mathrm{L}} \in \Theta_{1}, \forall \boldsymbol{p}_{\mathrm{G}} \in \Theta_{2}, \forall \boldsymbol{p}_{\mathrm{DG}} \in \Theta_{4}
\end{gathered}
$$

Solve (B-4) with fixed $\boldsymbol{x}_{L}$ as $\boldsymbol{x}_{L}^{z}$ and get the optimal result of $\boldsymbol{P}_{\mathrm{B}}, \boldsymbol{p}_{\mathrm{G}}$ and $\boldsymbol{p}_{\mathrm{DG}}$ as $\boldsymbol{P}_{\mathrm{B}}^{z+1}, \boldsymbol{p}_{\mathrm{G}}^{z+1}$ and $\boldsymbol{p}_{\mathrm{DG}}^{z+1}$. Then, formula (B-5) can be obtained.

$$
\begin{array}{r}
\operatorname{Max} F\left(\boldsymbol{x}_{\mathrm{L}}, \boldsymbol{p}_{\mathrm{G}}, \boldsymbol{P}_{\mathrm{B}}, \boldsymbol{p}_{\mathrm{DG}}\right) \\
\text { s.t. } \quad \boldsymbol{x}_{\mathrm{L}}=\boldsymbol{x}_{\mathrm{L}}^{z}, \boldsymbol{p}_{\mathrm{G}} \in \Theta_{2}, \boldsymbol{P}_{\mathrm{B}}=\boldsymbol{P}_{\mathrm{B}}^{z}, \boldsymbol{p}_{\mathrm{DG}} \in \Theta_{4} \\
F\left(\boldsymbol{x}_{\mathrm{L}}^{z}, \boldsymbol{p}_{\mathrm{G}}^{z+1}, \boldsymbol{P}_{\mathrm{B}}^{z+1}, \boldsymbol{p}_{\mathrm{DG}}^{z+1}\right) \geq F\left(\boldsymbol{x}_{\mathrm{L}}^{z}, \boldsymbol{p}_{\mathrm{G}}, \boldsymbol{P}_{\mathrm{B}}, \boldsymbol{p}_{\mathrm{DG}}\right) \\
\forall \boldsymbol{p}_{\mathrm{G}} \in \Theta_{2}, \forall \boldsymbol{P}_{\mathrm{B}} \in \Theta_{3}, \forall \boldsymbol{p}_{\mathrm{DG}} \in \Theta_{4}
\end{array}
$$

Due to (B-3), (B-6) can be obtained when $z=z+1$.

$$
\begin{array}{r}
F\left(\boldsymbol{x}_{\mathrm{L}}^{z+1}, \boldsymbol{p}_{\mathrm{G}}^{z+1}, \boldsymbol{P}_{\mathrm{B}}^{z+1}, \boldsymbol{p}_{\mathrm{DG}}^{z+1}\right) \geq F\left(\boldsymbol{x}_{\mathrm{L}}, \boldsymbol{p}_{\mathrm{G}}, \boldsymbol{P}_{\mathrm{B}}^{z+1}, \boldsymbol{p}_{\mathrm{DG}}\right) \\
\forall \boldsymbol{x}_{\mathrm{L}} \in \Theta_{1}, \forall \boldsymbol{p}_{\mathrm{G}} \in \Theta_{2}, \forall \boldsymbol{p}_{\mathrm{DG}} \in \Theta_{4}
\end{array}
$$

Thus, there is formula (B-7): 


$$
F\left(\boldsymbol{x}_{\mathrm{L}}^{z+1}, \boldsymbol{p}_{\mathrm{G}}^{z+1}, \boldsymbol{P}_{\mathrm{B}}^{z+1}, \boldsymbol{p}_{\mathrm{DG}}^{z+1}\right) \geq F\left(\boldsymbol{x}_{\mathrm{L}}^{z}, \boldsymbol{p}_{\mathrm{G}}^{z+1}, \boldsymbol{P}_{\mathrm{B}}^{z+1}, \boldsymbol{p}_{\mathrm{DG}}^{z+1}\right)
$$

According to (B-5), (B-8) is obtained.

$$
\begin{array}{r}
F\left(\boldsymbol{x}_{\mathrm{L}}^{z+1}, \boldsymbol{p}_{\mathrm{G}}^{z+1}, \boldsymbol{P}_{\mathrm{B}}^{z+1}, \boldsymbol{p}_{\mathrm{DG}}^{z+1}\right) \geq F\left(\boldsymbol{x}_{\mathrm{L}}^{z}, \boldsymbol{p}_{\mathrm{G}}, \boldsymbol{P}_{\mathrm{B}}, \boldsymbol{p}_{\mathrm{DG}}\right) \\
\forall \boldsymbol{p}_{\mathrm{G}} \in \Theta_{2}, \forall \boldsymbol{P}_{\mathrm{B}} \in \Theta_{3}, \forall \boldsymbol{p}_{\mathrm{DG}} \in \Theta_{4}
\end{array}
$$

Finally, formula (B-9) is proved.

$$
F\left(\boldsymbol{x}_{\mathrm{L}}^{z+1}, \boldsymbol{p}_{\mathrm{G}}^{z+1}, \boldsymbol{P}_{\mathrm{B}}^{z+1}, \boldsymbol{p}_{\mathrm{DG}}^{z+1}\right) \geq F\left(\boldsymbol{x}_{\mathrm{L}}^{z}, \boldsymbol{p}_{\mathrm{G}}^{z}, \boldsymbol{P}_{\mathrm{B}}^{z}, \boldsymbol{p}_{\mathrm{DG}}^{z}\right)
$$

(B-9) presents that $F\left(\boldsymbol{x}_{\mathrm{L}}^{z}, \boldsymbol{p}_{\mathrm{G}}^{z}, \boldsymbol{P}_{\mathrm{B}}^{z}, \boldsymbol{p}_{\mathrm{DG}}^{z}\right)$ is an increasing function. Note that the value of $F$ has an upper bound $F_{\max }^{*}$. Therefore, the TL_ATC method must converge. The convergence of the TL_ATC method ensures that a feasible decentralized strategy can be obtained. Since the problem is non-convex, the result may converge to a local maximum. However, because the three loop iteration part (step 2-4 in Fig. 5) of the TL_ATC method starts with the shared variables with the global optimal values of the convex models (step 1 in Fig. 5), the proposed method can provide a result with an acceptable optimization gap.

\begin{tabular}{|c|c|c|c|c|c|c|c|c|}
\hline SYS & Unit & $\begin{array}{c}\mathrm{P} \\
(\mathrm{MW})\end{array}$ & $\begin{array}{c}\mathrm{P}_{\max } \\
\text { (MW) }\end{array}$ & $\begin{array}{c}r \\
(\mathrm{MW} / \mathrm{h})\end{array}$ & SYS & DG & $\begin{array}{c}\mathrm{P}_{\min } \\
(\mathrm{MW})\end{array}$ & $\begin{array}{c}\mathrm{P}_{\max } \\
\text { (MW) }\end{array}$ \\
\hline \multirow{4}{*}{$\mathrm{TS}$} & 1 & 50 & 220 & 50 & \multirow{2}{*}{ ADG1 } & 1 & 0 & 15 \\
\hline & 2 & 30 & 100 & 50 & & 2 & 0 & 18 \\
\hline & 3 & 20 & 25 & 20 & \multirow{2}{*}{ ADG2 } & 1 & 5 & 28 \\
\hline & & & & & & 2 & 0 & 19 \\
\hline
\end{tabular}

\section{APPENDIX C}

TABLE III

GENERATOR AND DG DATA

TABLE IV

TRANSMISSION NETWORK DATA

\begin{tabular}{cccc}
\hline \hline From Bus & To Bus & X(p.u.) & Flow limit(MW) \\
\hline 1 & 2 & 0.170 & 200 \\
\hline 1 & 4 & 0.258 & 200 \\
\hline 2 & 3 & 0.037 & 190 \\
\hline 2 & 4 & 0.197 & 200 \\
\hline 3 & 6 & 0.018 & 180 \\
\hline 4 & 5 & 0.037 & 190 \\
\hline 5 & 6 & 0.140 & 180 \\
\hline \hline
\end{tabular}

TABLE V

DISTRIBUTION NETWORK DATA

\begin{tabular}{cccc|cccc}
\hline \hline \multicolumn{3}{c|}{ ADG1 } & \multicolumn{4}{c}{ ADG2 } \\
\hline From & To & X (p.u.) & $\begin{array}{c}\text { Flow } \\
\text { limit }\end{array}$ & From & To & X (p.u.) & $\begin{array}{c}\text { Flow } \\
\text { limit }\end{array}$ \\
\hline B3 & 1 & 0.2 & 60 & B4 & 1 & 0.2 & 70 \\
\hline 1 & 2 & 0.19 & 60 & 1 & 2 & 0.15 & 70 \\
\hline 2 & 3 & 0.21 & 30 & 2 & 3 & 0.2 & 90 \\
\hline 2 & 7 & 0.21 & 30 & 3 & 4 & 0.16 & 70 \\
\hline 3 & 4 & 0.20 & 40 & 4 & 5 & 0.18 & 40 \\
\hline 4 & 5 & 0.18 & 20 & 4 & 6 & 0.18 & 50 \\
\hline 4 & 6 & 0.18 & 30 & 6 & 7 & 0.16 & 40 \\
\hline 7 & 8 & 0.19 & 20 & & & & \\
\hline 8 & 9 & 0.19 & 20 & & & & \\
\hline \hline
\end{tabular}

\begin{tabular}{|c|c|c|c|c|c|c|c|c|c|}
\hline TS(MW) & 0 & 0 & 0 & 0 & 100 & 0 & & & \\
\hline ADG1(MW) & 0 & 7.5 & 12 & 0 & 0 & 9.5 & 12.5 & 0 & 8.5 \\
\hline ADG2(MW) & 0 & 0 & 33 & 0 & 18 & 28 & 21 & & \\
\hline
\end{tabular}

TABLE VI

LOAD DATA

$\begin{array}{llllllllll}\text { SYS } & \text { B1 } & \text { B2 } & \text { B3 } & \text { B4 } & \text { B5 } & \text { B6 } & \text { B7 } & \text { B8 } & \text { B9 }\end{array}$

TABLE VII

GENERATOR DATA

\begin{tabular}{ccccccc}
\hline \hline Bus & $\mathrm{P}$ & $\mathrm{Q}_{\max }$ & $\mathrm{Q}_{\min }$ & $\mathrm{P}_{\max }$ & $\mathrm{P}_{\min }$ & $r(\mathrm{MW} / \mathrm{h})$ \\
\hline 10 & 305.52 & 200 & -147 & 550 & 0 & 200 \\
\hline 12 & 39.24 & 120 & -35 & 185 & 0 & 90 \\
\hline 25 & 198.05 & 140 & -47 & 320 & 0 & 180 \\
\hline 26 & 141.56 & 1000 & -1000 & 414 & 0 & 180 \\
\hline 31 & 0 & 300 & -300 & 107 & 0 & 60 \\
\hline 46 & 112.78 & 100 & -100 & 119 & 0 & 60 \\
\hline 49 & 215.87 & 210 & -85 & 304 & 0 & 180 \\
\hline 54 & 41.62 & 300 & -300 & 148 & 0 & 60 \\
\hline 59 & 188.43 & 180 & -60 & 255 & 0 & 90 \\
\hline 61 & 185.15 & 300 & -100 & 260 & 0 & 90 \\
\hline 65 & 202.59 & 200 & -67 & 491 & 0 & 200 \\
\hline 66 & 202.83 & 200 & -67 & 492 & 0 & 200 \\
\hline 69 & 257.03 & 999 & -300 & 805.2 & 0 & 420 \\
\hline 80 & 140.23 & 280 & -165 & 577 & 0 & 200 \\
\hline 87 & 0 & 1000 & -100 & 104 & 0 & 60 \\
\hline 89 & 309.12 & 300 & -210 & -707 & 0 & -100 \\
\hline 100 & 243.48 & 155 & -50 & 352 & 0 & 180 \\
\hline 103 & 0 & 40 & -15 & 140 & 0 & -150 \\
\hline 111 & 0 & 1000 & -100 & 136 & 0 & 60 \\
\hline \hline
\end{tabular}

TABLE VIII

TS BOUNDARY BUSES AND THE CONNECTED DS TOTAL LOAD AMOUNT

TS Bus [7 181920272829333435395357587074757677 $8490102104105106107108109112118]$

Load amount [49604548 71 47 34 43 5933 3743 324266 68 61 41 (MW) $163553831435032486853]$

TABLE XIV

DG OUTPUT DATA IN DISTRIBUTION SYSTEM

\begin{tabular}{ccc}
\hline \hline DS bus & $\mathrm{P}_{\min }(\mathrm{MW})$ & $\mathrm{P}_{\max }(\mathrm{MW})$ \\
\hline 6 & 0 & 100 \\
\hline 18 & 0 & 70 \\
\hline 33 & 0 & 30 \\
\hline \hline
\end{tabular}

The input data used in the T6D2 system are listed in Tables III-VI. The T118D30 system has IEEE-118 transmission system with 30 ADSs. The ADSs are the modified IEEE-33 systems with five DGs shown in Table XIV. The generator data are presented in Table VII. Table VIII shows the TS boundary buses which connect the TS and 30 DSs. The corresponding load amount is the total load amount of the connected IEEE-33 system. The load amount of each load block in the ADS is proportionally distributed according to the total amount in Table VIII and the initial IEEE-33 system load amount, e.g., the total amount in Table VIII is $a$, the initial DS total load amount is $b$ and the DS load amount connected to one bus is $c$, then, the used load amount is $b / a^{*} c$. All the other data follows the standard IEEE-118 system and IEEE-33 system.

\section{REFERENCES}

[1] M. Adibi, P. Clelland, L. Fink, H. Happ, R. Kafka, J. Raine, D. Scheurer, and F. Trefny, "Power System Restoration-A Task Force Report," IEEE Trans. Power Syst., vol. 2, no. 2, pp. 271-277, 2011.

[2] M. Panteli, D. N. Trakas, P. Mancarella, and N. D. Hatziargyriou, "Power Systems Resilience Assessment: Hardening and Smart Operational 
Enhancement Strategies," Proc. IEEE, vol. 105, no. 7, pp. 1202-1213, 2017.

[3] M. M. Adibi and L. H. Fink, "Overcoming restoration challenges associated with major power system disturbances-restoration from cascading failures," IEEE Power Energy Mag., vol., no. 5, pp. 68-77, 2006.

[4] MISO power system restoration Plan, 2016, [Online]. Available: https://www.misoenergy.org/Library/Repository/Procedure/RTO-PSR001 MISO Power System Restoration Plan Manual Volume I Version9.1.pdf

[5] FERC/NERC Staff Report on the September 8, 2011 Blackout, [Online]. Available: https://www.ferc.gov/legal/staff-reports/04-27-2012-fercnerc-report.pdf

[6] C. Chen, J. Wang, and D. Ton, "Modernizing Distribution System Restoration to Active Grid Resiliency Against Extreme Weather Events: An Integration Solution," Proc. IEEE, vol.105, no. 7, pp.1267-1288, 2017.

[7] H. Jia, W. Qi, Z. Liu, B. Wang, Y. Zeng and T. Xu. "Hierarchical Risk Assessment of Transmission System Considering the Influence of Active Distribution Network" IEEE Trans. Power Syst., vol. 30, no. 2, pp. 1084-1093, 2015.

[8] A. Zegers and H. Brunner, "TSO-DSO interaction: An Overview of current interaction between transmission and distribution system operators and an assessment of their cooperation in Smart Grids," Sep. 2014 [Online]. Available: http://www.ieaisgan.org/index.php?r=home\&c $=5 / 378$

[9] ACER report. Energy Regulation: A Bridge to 2025, 2014, [Online]. Available: http://www.acer.europa.eu/official documents/acts of the agency/sd052005/supporting\%20document $\% 20$ to\%20acer\%20recomme ndation $\% 2005-2014 \% 20-\% 20 \% 20$ energy $\% 20$ regulation $\% 20 \mathrm{a} \% 20 \mathrm{bridg}$ e\%20to\%202025\%20conclusions\%20paper.pdf

[10] ENTSO report. General Guidelines for Reinforcing the Cooperation Between TSOs and DSOs, 2015, [Online]. Available: https://www.entsoe.eu/Documents/Publications/Position\%20papers\%20 and\%20reports/entsoe_pp_TSO-DSO_web.pdf

[11] A. Kargarian and Y. Fu, "System of Systems Based SecurityConstrained Unit Commitment Incorporating Active Distribution Grids," IEEE Trans. Power Syst., vol. 29, no. 5, pp. 2489-2498, 2014.

[12] Z. Li, Q. Guo, H. Sun, and J. Wang, "Coordinated Economic Dispatch of Coupled Transmission and Distribution Systems Using Heterogeneous Decomposition," IEEE Trans. Power Syst., vol. 31, no. 6, pp. 4817-1830, 2016.

[13] W. Liu, Z, Lin, F. Wen, and G. Ledwich, "A wide area monitoring system based load restoration method," IEEE Trans. Power Syst., vol. 28, no.2, pp. 2025-2034, 2013.

[14] W. Sun, C.-C. Liu, and L. Zhang, "Optimal start-up strategy for bulk power system restoration," IEEE Trans. Power Syst., vol. 26, no. 3, pp. 1357-1366, Aug. 2011.

[15] A. Gholami and F. Aminifar, "A Hierarchical Response-Based Approach to the Load Restoration Problem," IEEE Trans. Smart Grid, no. 99, pp. $1-10,2015$.

[16] A. Arif, Z. Wang, J. Wang and C. Chen, "Power Distribution System Outage Management with Co-Optimization of Repairs, Reconfiguration, and DG Dispatch," IEEE Trans. Smart Grid, to be published.

[17] H. Yeh, D. F. Gayme, and S. H. Low, "Adaptive VAR Control for Distribution Circuit With Photovoltaic Generators," IEEE Trans. Power Syst., vol. 27, no. 3, pp 1656-1663, 2012.

[18] C. Lin, W. Wu, B. Zhang and et al, "Decentralized Reactive Power Optimization Method for Transmission and Distribution Networks Accommodating Large-Scale DG Integration" IEEE Trans. Sustain. Energy, vol. 8, no. 1, pp. 363-373, 2017.

[19] J. Joo and M. D. Ilic, "Multi-layered optimization of demand resources using Lagrange dual decomposition," IEEE Trans. Smart Grid, vol. 4, no. 4, pp. 2081-2088, Dec. 2013.

[20] A. Kargarian, J. Mohammadi, J. Guo and et al, "Toward Distributed/Decentralized DC Optimal Power Flow Implementation in Future Electric Power Systems," IEEE Trans. Smart Grid, vol. 9, no. 4, pp. 2574-2593, 2018.

[21] A. Kargarian, Y. Fu and Z. Li, "Distributed Security-Constrained Unit Commitment for Large-Scale Power Systems," IEEE Trans. Power Syst., vol. 30, no. 4, pp. 1925-1936, 2015.

[22] Z. Li, M. Shahidehpour, W. Wu, B. Zeng, B. Zhang and W. Zheng, "Decentralized Multiarea Robust Generation Unit and tie-line scheduling under Wind Power Uncertainty," IEEE Trans. Sustain. Energy, vol. 6, no. 4, pp. 1377-1388, 2015.
[23] A. Ahmadi-Khatir, A. J. Conejo, and R. Cherkaoui, "Multi-area unit scheduling and reserve allocation under wind power uncertainty," IEEE Trans. Power Syst., vol. 29, no. 4, pp. 1701-1710, 2014.

[24] A. G. Bakirtzis and P. N. Biskas, "A Decentralized Solution to the DCOPF of Interconnected Power Systems," IEEE Trans. Power Syst., vol. 18, no. 3, pp. 1007-1013, 2003.

[25] Z. Y. and M. R. Hesamzadeh, "Hierarchical coordination of TSO-DSO economic dispatch considering large-scale integration of distributed energy resources," Applied Energy, vol. 195, pp. 600-615, 2017.

[26] Z. Lin, F. Wen, and Y. Xue, "A Restorative Self-Healing Algorithm for Transmission Systems Based on Complex Network Theory," IEEE Trans. Smart Grid, vol. 7, no. 4, pp. 2154-2162, 2016.

[27] L. H. Fink, K. L. Liou and C. C. Liu, "From Generic Restoration Actions to Specific Restoration Strategies," IEEE Trans. Power Syst., vol. 10, no. 2, pp. 745-752, 1995.

[28] J. Feltes and C. G. Moran, "Down, but not out: a brief overview of restoration issues," IEEE Power and Energy Mag., vol. 12, no. 1, pp. 34-43, 2014.

[29] ENTSO report. Towards smarter grids: Developing TSO and DSO roles and interactions for the benefit of consumers, 2015, [Online]. Available: https://www.entsoe.eu/Documents/Publications/Position\%20papers\%20 and\%20reports/150303_ENTSO-E_Position_Paper_TSODSO_interaction.pdf

[30] Y. Hou, C. C. Liu, K. Sun, P. Zhang, S. Liu, and D. Mizumura, "Computation of Milestones for Decision Support During System Restoration," IEEE Trans. Power Syst., vol. 26, no. 3, pp. 1399-1409, Aug. 2011.

[31] G. Monique and S. Kim, "Lagrangean decomposition: A model yielding stronger Lagrangean bounds," Math. Program., vol. 39, no. 2, pp. 215$228,1987$.

[32] S. Tosserams, L. F. P. Etman, P. Y. Papalambros, and J. E. Rooda, “An augmented Lagrangian relaxation for analytical target cascading using the alternating directions method of multipliers," Struct. Multdisc. Optim., vol. 31, no. 3, pp. 176-189, 2006.

[33] M. M. Adibi, J. N. Borkoski, R. J. Kafka, et al., "Frequency response of prime movers during restoration," IEEE Trans. Power Syst, vol.14, no.2, pp. 751-756, May. 1999.

[34] C. Chen, J. Wang, and D. Ton, "Modernizing Distribution System Restoration to Active Grid Resiliency Against Extreme Weather Events: An Integration Solution," Proc. IEEE, vol.105, no. 7, pp.1267-1288, 2017.

[35] SmartGrid.gov. (May 2014). Recovery Act Smart Grid Programs: Project Information. [Online]. Available: https://www.smartgrid.gov/ recovery act/project information

[36] Y. Liu, R. Fan and V. Terzija, "Power system restoration: a Literature review from 2006 to 2016," J. Mod. Power syst. Clean energy, vol. 4, no. 3, pp. 332-341, 2016

[37] D. P. Bertsekas, Nonlinear Programming, 2nd ed. Belmont, MA, USA: Athena Scientific, 2003.

[38] N. F. Michelena, H. Park, and P. Y. Papalambros, "Convergence properties of analytical target cascading," AIAA J., vol. 41, no. 5, pp. 897-905, May 2003.

[39] C. Coffrin, P. Van Hentenryck, "A linear-programming approximation of AC power flows," INFORMS J. Comput., vol. 26, no. 4, pp. 718-734, Nov. 2014. 